\title{
Analysis of ARROW Waveguide based Microcantilever for Sensing Application
}

Asha K ( $\square$ asha.k@saividya.ac.in )

Sai Vidya Institute of Technology

Narayan Krishnaswamy

Sai Vidya Institute of Technology

N K Suryanarayana

Sai Vidya Institute of Technology

\section{Research Article}

Keywords: Microcantilever, 2.5D FDTD, Quality factor, Sensitivity, ARROW WAVEGUIDE

Posted Date: June 4th, 2021

DOI: https://doi.org/10.21203/rs.3.rs-579000/v1

License: (c) (1) This work is licensed under a Creative Commons Attribution 4.0 International License. Read Full License 


\title{
Analysis of ARROW Waveguide based Microcantilever for Sensing Application
}

\section{Asha K ${ }^{1}$, Narayan Krishnaswamy ${ }^{2,}$ and Suryanarayana $\mathbf{N}^{3}$}

\author{
${ }^{1}$ Research Scholar and Assistant Professor, Department of Electronics \& Communication Engineering, Sai \\ Vidya Institute of Technology, Bangalore, Affiliated to Visvesvaraya Technological University, Belagavi, India, \\ asha.k@saividya.ac.in \\ ${ }^{2}$ Dean R\&D, Professor, and SMIEE, Life-Member IEEE-HKN, Department of Electronics \& Communication \\ Engineering, Sai Vidya Institute of Technology, Bangalore, Affiliated to Visvesvaraya Technological \\ University, Belagavi, India, narayank101@gmail.com

\footnotetext{
${ }^{3}$ Research Scholar and Assistant Professor, Department of Electronics \& Communication Engineering, Sai
} \\ Vidya Institute of Technology, Bangalore, Affiliated to Visvesvaraya Technological University, Belagavi, India, \\ suryanarayana.nk@saividya.ac.in
}

\begin{abstract}
Analysis of microcantilever beam and ARROW microcantilever waveguides are presented in this work. The microcantilever is simulated by using the silicon nitride material. Electric voltage applied creates the deformation in the cantilever beam. The deformation leads to displacement of the beam. The displacement is due to bending of the cantilever tip. The integration of MEMS cantilever and ARROW waveguide results in the ARROW microcantilever waveguide. The ARROW microcantilever waveguide quality factor, electric filed intensity and sensitivity analysis are the three important parameters presented. The quality factor is obtained by varying the air gap distance between cantilever waveguide and output waveguide. Through this simulation using FDTD sensitivity up to $73.78 \mathrm{~nm} / \mathrm{RIU}$ has been achieved for the microcantilever arrow waveguide.
\end{abstract}

Keywords: Microcantilever, 2.5D FDTD, Quality factor, Sensitivity, ARROW WAVEGUIDE.

\section{Introduction}

Microcantilever devices are used for sensing application. The cantilever array biosensor designed has capability of detecting analyte sample. The design was developed mainly to improve the sensitivity of the cantilever-based sensor [1]. The material such as silicon [2], silicon nitride [12] and polymer materials are used for simulating and fabricating the cantilever. The mechanical property of the cantilever designed is very important. M-test was conducted to measure the mechanical property. The mathematical modeling tools such as finite difference, finite element method and energy method are used for the simulation [2].

Atomic force microscope (AFM) has microcantilever device in its architecture. The beam immersed in viscous fluid has a response. The measurement of the frequency response for the designed cantilever has application in atomic force microscopy [3]. The cantilever mainly used in AFM and excitation of the tip of cantilever using electrical signal is discussed. The sample surface topography can be scanned using multiple cantilever [4]. Another application of the cantilever is in optical waveguide. Nano mechanical transduces that has mechanical and optical models. The sensor developed has sensitivity of $5.7 \times 10^{-4} \mathrm{~nm}^{-1}$. The modelling method involved in the design was finite element method (FEM) [5]. Nanomechanical sensor also used for detection of femtogram mass using photo thermal actuation. A gold coated nano mechanical cantilever is used with frequency range from 1 to $10 \mathrm{MHz}$ [25].

Certain cantilever is used in environmental application as a sensor. The thermal sensors and humidity sensors are the example. The piezoresistive read out based integrated device was modelled. The model is promising tool for sensing application in liquid environment [6]. MEMS cantilever device are popular structure and they are used as a resonator. These structures are of small size, energy consumption is less and durable. The silicon based microcantilever has quality factor of 35 [7]. The electrostatically actuated device with deflection model is modeled and simulated using MEMS [8].

Gallium nitride microcantilever with vanadium oxide material used as thermal sensor. The variation of the temperature of cantilever device result in the sensitivity change and is used in sensing application [9]. The vanadium oxide is the piezoresistive material used in the sensor. The cantilever designed using polymer material, which has length greater than that of the width [10]. The shape of the cantilever varies from one application to other; the T shaped cantilever device is used as sensor. The analyte used for the experimental purpose was DNA chains [11]. The shapes of the cantilever are T-shape cantilever, slotted cantilever, paddle cantilever and triangular 
cantilever. The variation of the slot gives variation in the frequency value and quality factor value. Based on the gap length of waveguide from 0 to $40 \mu \mathrm{m}$ there is change in the quality factor from 0 to 800 [12].

The flow sensor cantilever waveguide is designed using polydimethylsiloxane (PDMS) material. The deflection of the cantilever was analyzed with experimental values [13]. The complete flow sensor with integrated microchannel and detection system is simulated. The software used in the simulation process is COMSOL Multiphysics [14]. PDMS polymer is very versatile material for microfabrication because of its properties for the medical application. The properties are biocompatibility of the material with other analyte sample and the chemical sample used in the modeling and transparency of the material. Array of cantilever beams are used imaging application in AFM. A non-linear system is developed for the scanning that has application in AFM such as cutting, pushing and lithography [15]. The laser bending of the cantilever waveguide was fabricated using silicon material. The main purpose is for parallel sensing of the position. The dimension of the device reported was $110 \times 13 \times 0.6 \mu \mathrm{m}$ respectively length width and thickness of the cantilever [16].

A chemical sensor using MEMS concept and position sensitive detector (PSD) are designed using the piezoelectric material for sensing purpose. Also simulated the multi-layer cantilever device [17]. Chemical vapor deposition (CVD) is used in the fabrication of the optical waveguide cantilever sensor. The two important CVD methods are low pressure CVD (LPCVD) and plasma enhanced CVD (PECVD). The beam propagation method (BPM) was the mathematical modeling method used to find the nanomechanical forces in the cantilever [18]. The fabricated silicon dioxide on silicon substrate based optical waveguide cantilever has actuated by light. This article shows that there is an exponential decay in the power output for applied power input at the cantilever structure. [19]. The design consideration discussed are dynamic analysis of the cantilever, lumped model analysis and stability analysis [20]. These electrostatically actuated microcantilever used as a bio mass sensor. These micro structures attached with the carbon nano tube (CNT). The single walled CNT as a sensor was explored [21].

The microcantilever sensor are also used detection of the variation in the $\mathrm{pH}$ values in the chemicals. The material used for designing are silicon nitride and silicon dioxide [22]. In electro mechanical device the calibration is very important. Reliable calibration is the main concentration in the Mishra et.al. paper. equipartition theorem and AFM are used for the purpose of calibration [23]. The microcantilever structure with grating is fabricated using two materials in the mid-IR range $(3-11 \mu \mathrm{m})$ to overcome the spectral bandwidth issues problem. The model enhances the sensitivity and transmission [24]. The micro milling and molding methods were used for the fabrication of the microcantilever beam [26]. The cantilever biosensor based on the adsorption method was reported. Proteins samples adsorption process on the functionalized cantilever surface results in the bend in the cantilever [27]. The analysis of nonlinear modes when the cantilever excitation was done using the invariant techniques with amplitude vibration [28]. The main concept used in major design and analysis of the cantilever is the optical readout method. The single mode waveguides are suitable for the optical read out method. The sensitivity of the sensor with air and liquid both discussed [29]

The first section is the introduction to the cantilever based micro nano devices. Second section is modeling of cantilever beam and reflecting optical waveguide (ARROW) microcantilever structure. The materials used for both the structure designing is discussed. The mechanical and optical modeling of the cantilever is explained. The section three is mainly the highlights on the results obtained for cantilever beam and ARROW microcantilever waveguide. The quality factor is also discussed. The section four is discussion on the electric filed intensity modeling of the ARROW cantilever and also on the sensitivity analysis of the ARROW microcantilever waveguide.

\section{Design of cantilever beam and cantilever ARROW waveguide.}

In this section we discuss the cantilever beam and anti-resonant reflecting optical waveguide (ARROW) cantilever structure. The ARROW devices are single mode, low loss and a good light confinement device used in sensing application [30]. The optical life confinement of the ARROW waveguide using 2.5D FDTD method is discussed. The ARROW waveguide used is B type where in the core and second cladding refractive are greater than that of the first cladding. This design is advantageous compared to the conventional or ARROW-A type waveguide. The ARROW device has two variation namely, hollow core ARROW and liquid core ARROW. In this section we discuss the modeling of the cantilever beam and ARROW-B cantilever waveguide. Further in discussion ARROW-B waveguide is simply denoted as ARROW waveguide.

\subsection{Modeling of Microcantilever Beam}

The cantilever has mechanical characteristics such as radius, stress, force, strain and momentum etc. The sensitivity of the microcantilever varies depending on the Poisson's ratio and Young's modulus. The basic characteristics of the cantilever are given below. The stress $(\sigma)$ is represented in equation (1).

$$
\sigma=\frac{F}{A}
$$

The strain $(\varepsilon)$ is given in equation $(2)$ 


$$
\varepsilon=\frac{\Delta L}{L}
$$

Young's modulus (E) is the stiffness of the material is given by equation (3).

$$
E=\frac{\sigma}{\varepsilon}
$$

Here $\mathrm{F}$ is the force applied and $\mathrm{A}$ is the area of cross section of the cantilever. $\Delta L$ is the change in the length and Length of the cantilever is L. The micro cantilever beam is shown in figure 1. The cantilever has $300 \mu \mathrm{m}$ length, width $20 \mu \mathrm{m}$ and thickness is $2 \mu \mathrm{m}$. The beam is firmly fixed at one side of cantilever and it freely moves at the other side of the cantilever.

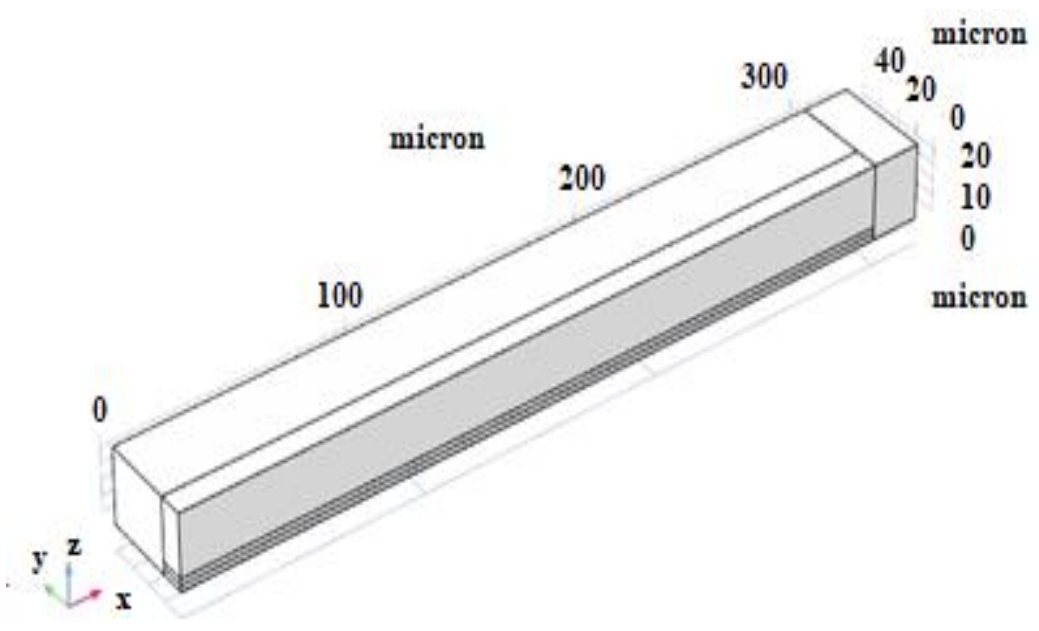

Figure 1. Microcantilever Beam

The $2 \mathrm{D}$ view of the cantilever is shown in figure 2 . The beam is located in the air-filled cabin. The geometry of the cantilever in the YZ plane is depicted. The insulated chamber is electrically isolated and electrode is grounded. The shown cantilever is fixed at $\mathrm{x}=0$. and has dimension of $300 \times 40 \times 20$ length, width and thickness respectively.

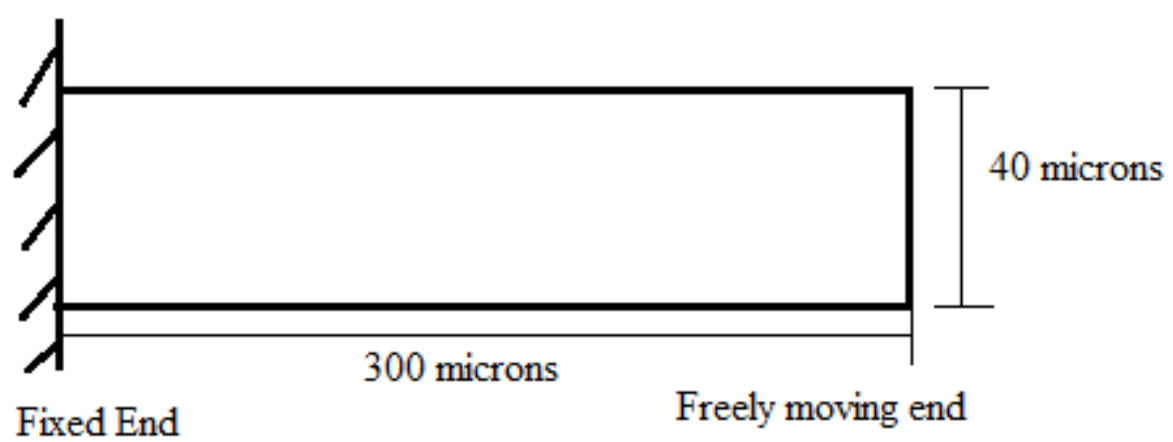

Figure 2. Two-dimensional view of the microcantilever beam

The common material used for MEMS based devices are silicon nitride and silicon material. The silicon nitride material property and dimension as shown in table 1 . The Young's modulus is quantity that shows the material with stand capability. It is also represented in terms of stress and strain. The Poisson's ratio of the material depends on the Poisson effect. In Poisson phenomenon, the material has tendency to extend in the direction perpendicular to its direction path. The density of the silicon nitride is mass per unit volume. The pressure and temperature change the material property of silicon nitride material. 
Table 1. Silicon Nitride material property

\begin{tabular}{ccc}
\hline SL No. & Parameter & Values \\
\hline 1 & Young's Modulus & $130 \mathrm{GPa}$ \\
2 & Poisson's ratio & 0.25 \\
3 & Density & $2800 \mathrm{~kg} / \mathrm{m}^{3}$ \\
4 & Width & $40 \mu \mathrm{m}$ \\
5 & length & $300 \mu \mathrm{m}$ \\
6 & Thickness & $20 \mu \mathrm{m}$ \\
\hline
\end{tabular}

The figure 3 shows the rectangular meshing used in the design. This is the simple meshing method to find the solution by dividing the geometry into smaller areas. Finite element method uses meshing concept to decrease the degree of freedom.

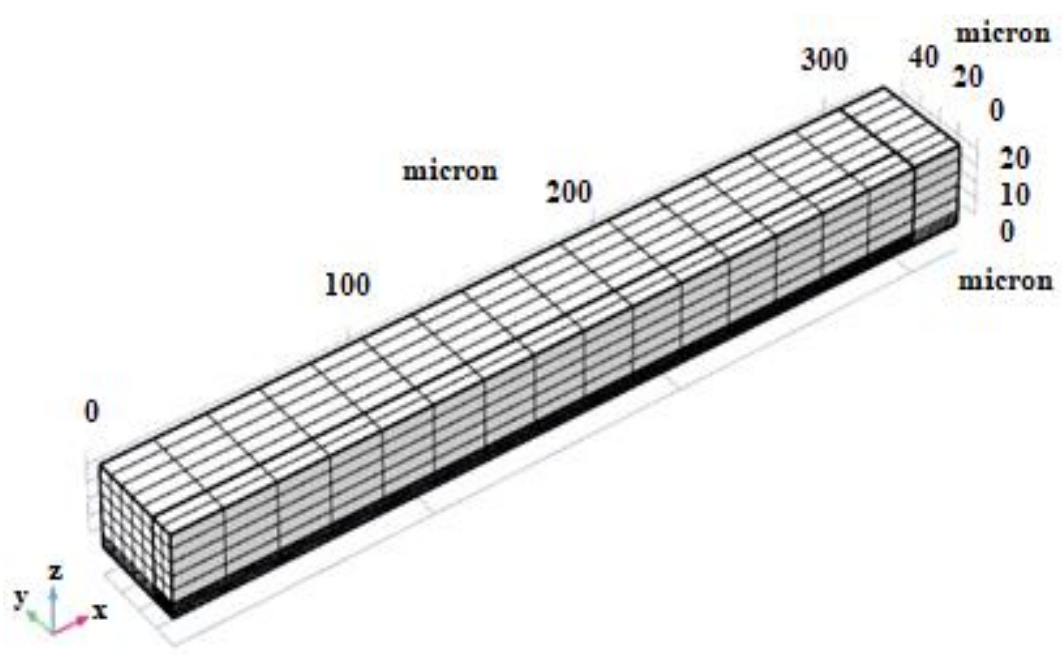

Figure 3. Meshing of microcantilever beam

\subsection{Microcantilever ARROW waveguide}

In this section we discuss the mechanical modeling of the cantilever waveguide. The concept of ARROW waveguide and its concepts in the cantilever waveguide and optical modeling of the cantilever waveguide.

\subsubsection{Mechanical Model}

The bending of the cantilever is characterized by the functional area. The interaction of the molecules on the surface of the cantilever waveguide leads to surface stress $\Delta \delta$, this intern leads to deflection in the waveguide and is given in the equation (4) [5].

$$
\Delta y \approx \frac{3 k(1-v)}{E}\left(\frac{l_{c}}{t_{c}}\right)^{2} \Delta \delta
$$

In above equation (4), $\mathrm{k}$ is constant depends on waveguide geometry and material. $v$ is the Poisson ratio, $l_{c}$ length of the cantilever, $t_{c}$ is the thickness of the cantilever and $\mathrm{E}$ is Young modulus.

\subsubsection{Concept of ARROW waveguide}

The ARROW B waveguide is incorporated in the simulation of the ARROW B microcantilever waveguide.

The ARROW waveguide is a five-layer device as shown in figure 4. The ARROW-B microcantilever has four regions of operation namely input waveguide region, second is cantilever waveguide, third region is the air gap and fourth region is the output waveguide. The selection of the dimension of the device is very important in the ARROW waveguide design. The selection was thickness of each layer is by considering the below equation $(5)$ and (6). The first cladding layer thickness $\left(t_{1}\right)$ is given by the below equation (5) [30]

$$
\mathrm{t}_{1}=\frac{\lambda}{4 n_{1}}\left[1-\left(\frac{n_{c}}{n_{1}}\right)^{2}+\left(\frac{\lambda}{2 \eta_{c} \mathrm{t} c}\right)^{2}\right]^{-1 / 2}(2 N+1)
$$


The second cladding layer has thickness $\left(t_{2}\right)$ half of the core thickness given in equation (6)

$$
\mathrm{t}_{2}=\frac{\mathrm{t}_{c}}{2}(2 \mathrm{~m}+1)
$$

here, $\mathrm{N}, \mathrm{m}$ are values $0,1,2$ etc.,

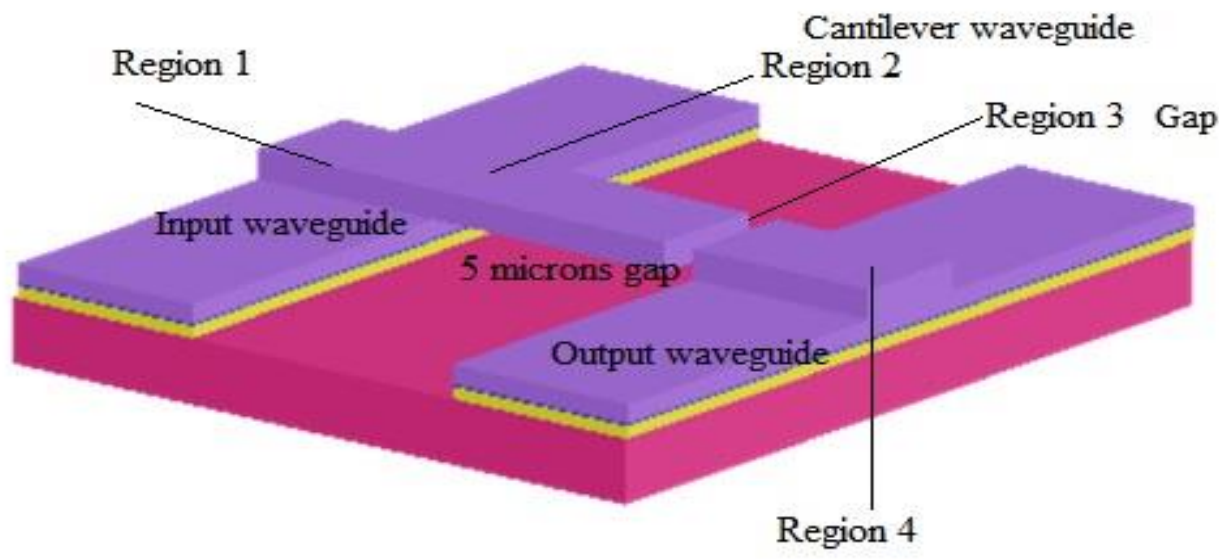

Figure 4. Microcantilever ARROW device

The refractive index values for the ARROW-B micro cantilever waveguide are tabulated in table2. Each layer material used and RI is given A bulk silicon substrate is considered, above which $2 \mu \mathrm{m}$ silicon nitride second cladding layer is deposited. A silicon dioxide layer of $0.12 \mu \mathrm{m}$ is fabricated and above the silicon dioxide layer we have $4 \mu \mathrm{m}$ silicon nitride layer is deposited. This completes the structure of ARROW microcantilever waveguide. The length, width and thickness of the structure is $40 \times 10 \times 10$ respectively.

Table 2. Refractive Index values of cantilever ARROW waveguide

\begin{tabular}{ccc}
\hline SL No. & Layers & RI \\
\hline 1 & Silicon Nitride Core & $2.00+\mathrm{j} 0$ \\
2 & Silicon dioxide First cladding & $1.46+\mathrm{j} 0$ \\
3 & Silicon Nitride Second cladding & $2.00+\mathrm{j} 0$ \\
4 & Silicon substrate & $3.85+\mathrm{j} 0.019$ \\
5 & Superstrate & $1+\mathrm{j} 0$ \\
\hline
\end{tabular}

The ARROW waveguide XY, XZ, YZ and complete view is shown in figure 5. The modal analysis carried for the ARROW -B microcantilever waveguide. The first cladding layer thickness is very small. The second cladding thickness is half of the core thickness. The transvers electric (TE) and transvers magnetic (TM) modes are present in the ARROW-B cantilever structures. The TE mode is used for the analysis purpose.
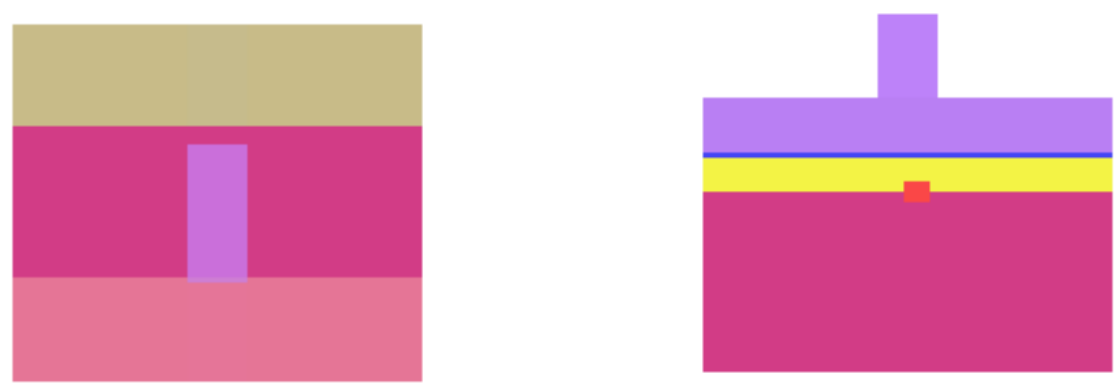

(a) XY view

(b) XZ view 


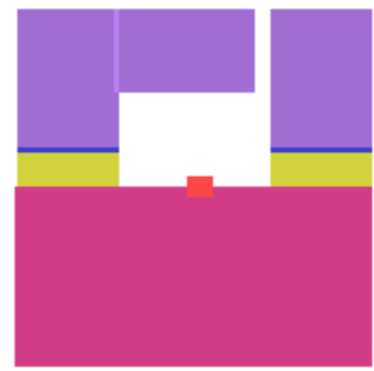

(c) YZ view

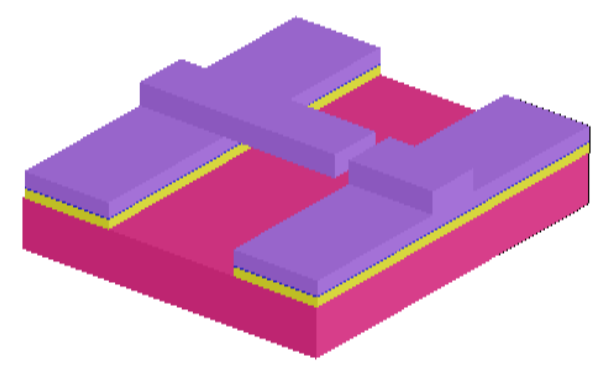

(d) Complete view of microcantilever

Figure 5. Directional view of Microcantilever ARROW device

\subsubsection{Optical method}

In optical method used for coupling of light from input waveguide to output waveguide in the ARROW-B cantilever. The light confinement inside the device is modelled by using optical modeling method. The center of the cantilever waveguide is designed using the below equation (7) [29].

$$
w(l)=w_{0}\left[\left(1+\frac{\lambda l}{\pi \omega_{0}^{2}}\right)^{2}\right]^{1 / 2}
$$

here, $w_{0}$ is the beam in the cantilever waveguide, wavelength is $\lambda$ and $l$ is the length of the waveguide. The light coupling efficiency is modeled by using equation (8) [29]

$$
\eta=\frac{\int_{-\infty}^{\infty} \int_{-\infty}^{\infty} \psi_{2}(x, y, l) \psi_{3}(x, y+\mathrm{d}) \mathrm{d} x \mathrm{~d} y}{\int_{-\infty}^{\infty} \int_{-\infty}^{\infty} \psi_{1}^{2}(x, y) \mathrm{d} x \mathrm{~d} y} X \frac{\int_{-\rho_{x} w_{\text {out }}}^{\rho_{x} w_{\text {out }}} \int_{-\rho_{y}}^{\rho_{y}} \psi_{2}(x, y, l) \psi_{4}(x, y+\mathrm{d}) \mathrm{d} x \mathrm{~d} y}{\int_{-\rho_{x} c t}^{\rho_{x} c t} \int_{-\rho_{y}}^{\rho_{y}} \psi_{2}^{2}(x, y) \mathrm{d} x \mathrm{~d} y}
$$

The mode profile of the input ARROW waveguide region is $\psi_{1}$, the for and second cantilever region, third air gap region and output waveguide region are having the mode profiles $\psi_{2}, \psi_{3}$ and $\psi_{4}$ respectively. $l$ is the length and cantilever deflection is $\mathrm{d}$. the half width of the output waveguide is $\rho_{x} w_{\text {out }} . \rho_{x} c t$ is the half width of the cantilever. The detailed structure showing the entire ARROW waveguide is represented in figure 6.

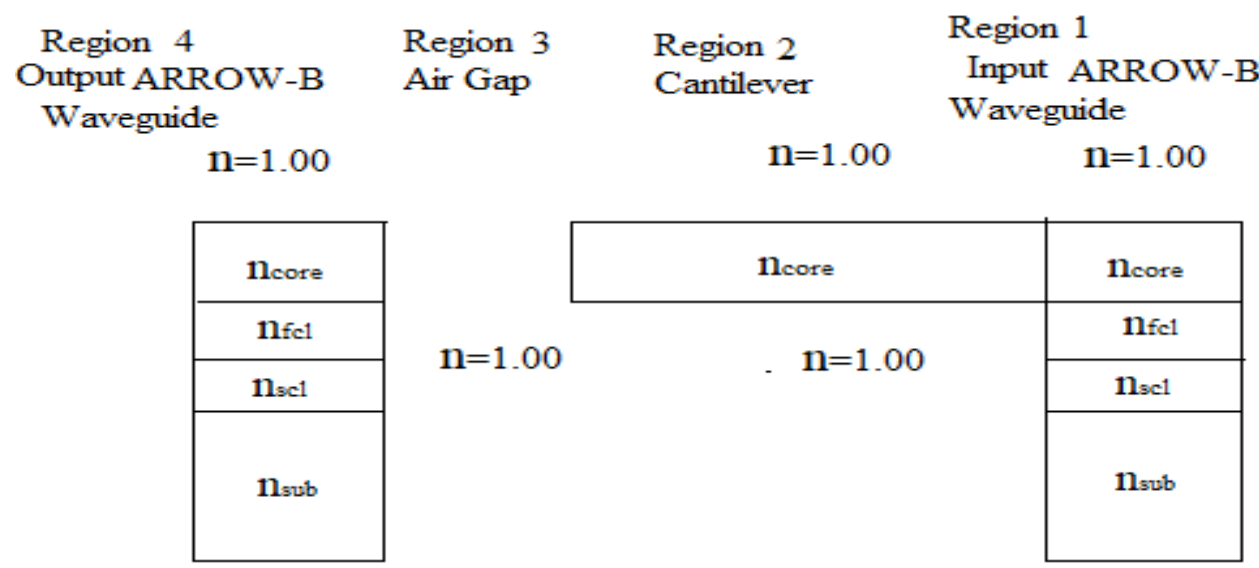

Figure 6. Regions of microcantilever ARROW waveguide 


\section{Results}

In this section we discuss about the electrical actuating of the cantilever waveguide and as well as the quality factor of the ARROW cantilever waveguide by varying the air gap distance in the structure.

\subsection{Results of electrostatically actuated microcantilever beam}

The result of the electrostatically actuated microcantilever beam is discussed in this section. The displacement, electric field and potentials and capacitance effect are the important parameter of discussion. The electrically applied voltage makes the deformation in the cantilever beam. The electrostatic field is given by Poisson theorem, which is shown in equation (9)

$$
\nabla \cdot(\varepsilon \nabla V)=0
$$

The mechanical and electrical interface is used in the design and modeling of the cantilever beam. The derivatives of the interface are considered as spatial coordinating system. The coupled physics is involved in the simulation and modeling of the cantilever. Finite element method is the mathematical modeling tool used. The electrical actuating of the microcantilever results in the positive feedback for the force applied. Electrostatic force causes the beam to bend. As it twists, the distance between it and the electrode which is grounded narrows. The voltage can be applied in regular intervals. The applied voltage when it reaches to particular value where in the beam collapses is called as pull-in-voltage. At this particular time the system becomes unstable.

The microcantilever is applied with voltage potential between the two electrodes. The boundaries surrounding the system is electrically insulated to avoid other losses in the system. The boundary conditions at the terminal are used to calculate the force density and is depicted in equation (10).

$$
F_{e s}=-0.5(E . D) n+(n . E) D
$$

In the design the $\mathrm{E}$ is the filed vector because of the electric field applied, displacement is $\mathrm{D}$ and $\mathrm{n}$ represents the outward vector of the boundary. Micro cantilever beam coordinate system is calculated using the Navier's equation. The microcantilever beam will be in equilibrium state. The electrical voltage applied results in the displacement of the beam. Figure 7 shows the displacement of the cantilever beam along the z-axis.

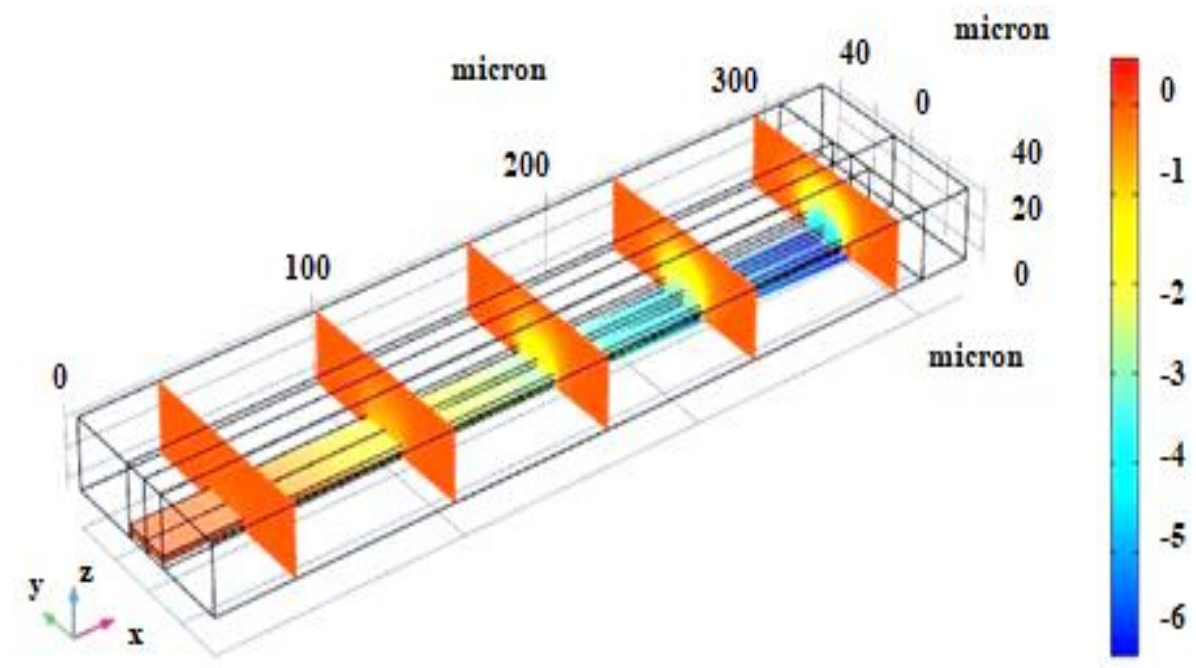

Figure 7. Displacement of the cantilever

The electric potential across the microcantilever beam is shown in figure 8 . 


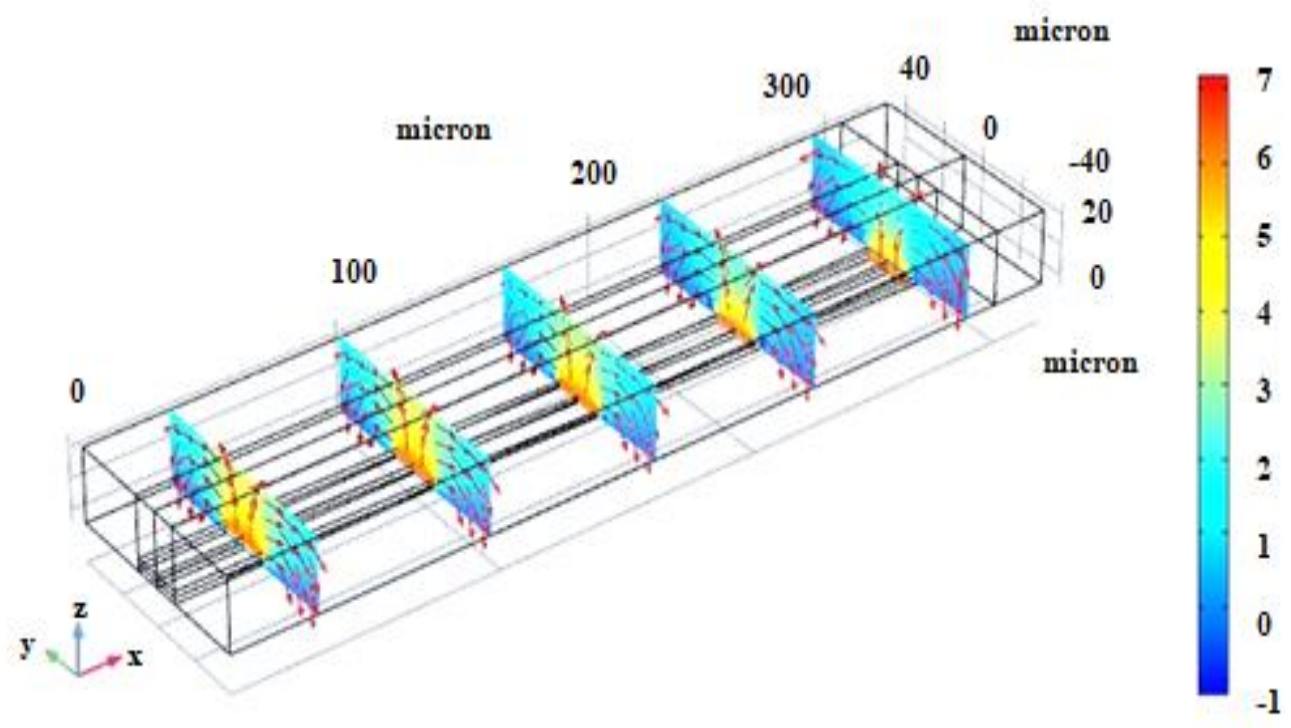

Figure 8. Electric field and potential of cantilever beam

The applied voltage on the silicon nitride microcantilever simulation leads the displacement in the cantilever along $\mathrm{z}$ direction is shown in figure 9. The shape of the cantilever for different applied voltage is shown.

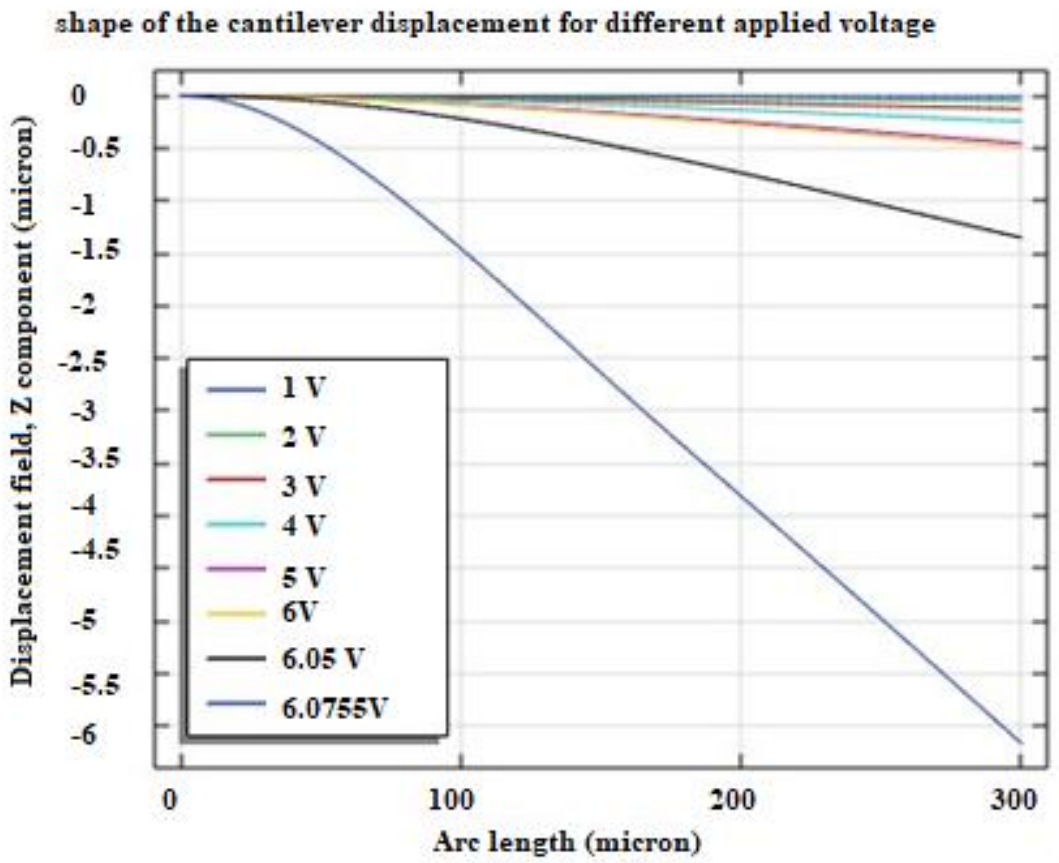

Figure 9. Cantilever Displacement versus arc length.

Figure 10 depicts the bending of the cantilever due to force acting on it. The displacement field orientation direction is in $\mathrm{z}$-axis. When voltage of $\mathrm{V} 0=1$ volt is applied, the measured displacement is zero. From voltage values of 2 volts to 5 volts the cantilever beam undergoes deformation. When the voltage is 6.0755 Volts i.e., when it reaches the pull-in voltage the cantilever based electrostatically actuated microcantilever collapses and becomes unstable. 


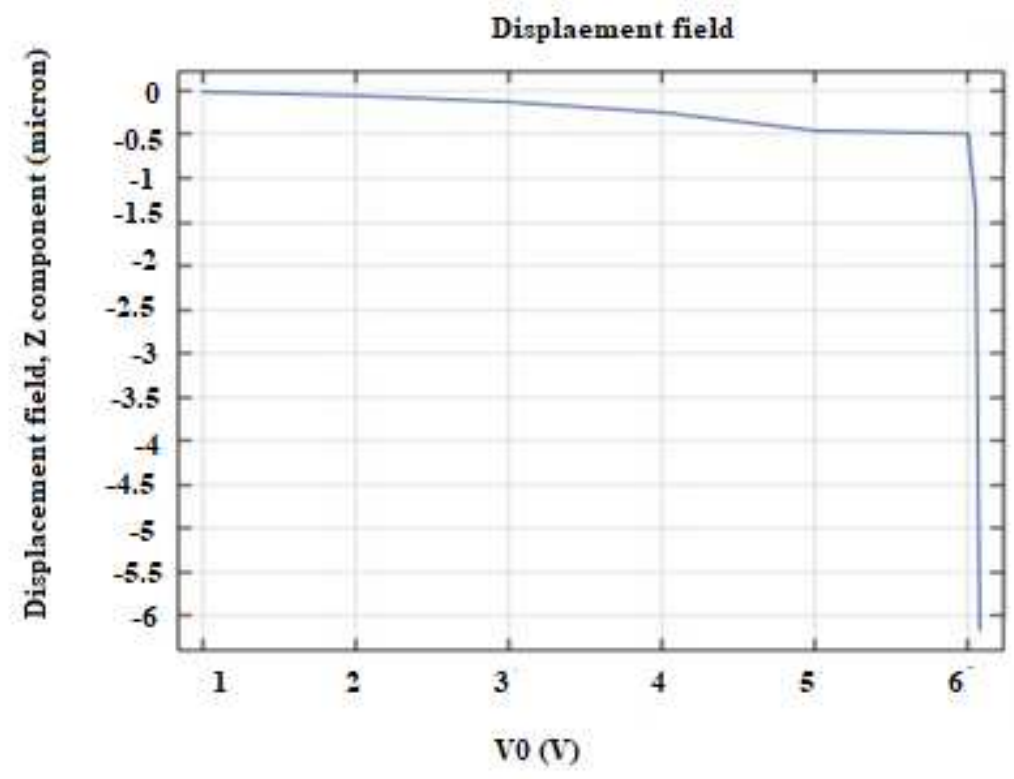

Figure 10. Electrical voltage applied versus Displacement.

The figure 11 depicts the microcantilever beam DC capacitance curve. The action is comparable to that of the parrel plate capacitance effect. Applied voltage makes the beam to soften. When softening occurs deformation continuous upon applying the voltage. Importance is to maintain the potential difference between the two electrodes in the microcantilever system modeled. As observed in the figure capacitance values are from $36 \mathrm{fF}$ to $62 \mathrm{fF}$.

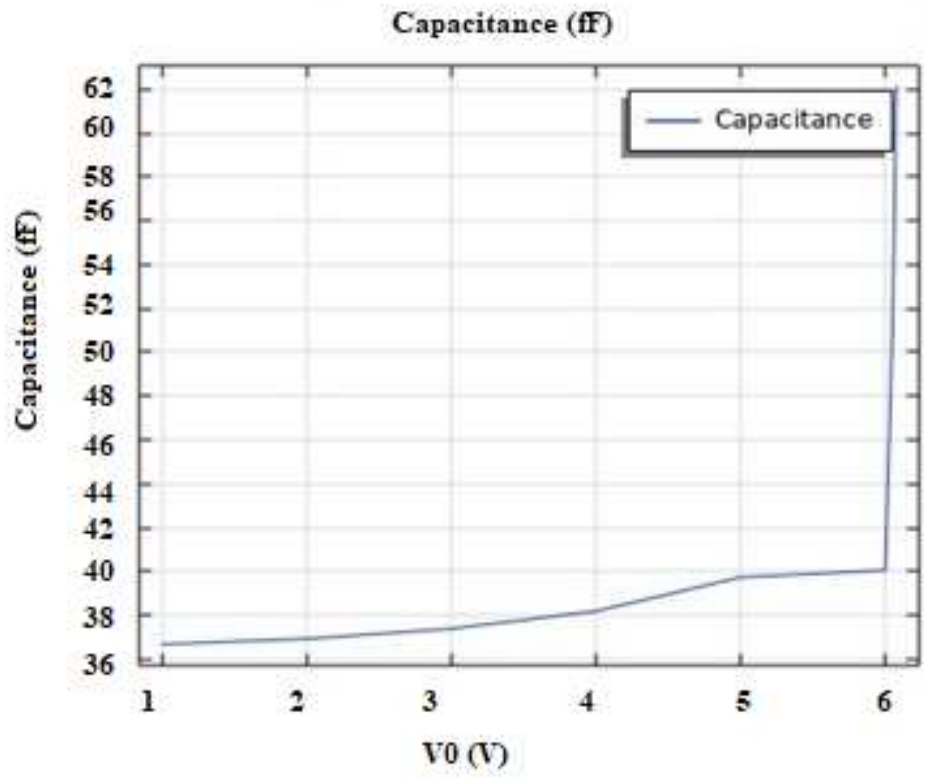

Figure 11. DC capacitance versus voltage applied

\subsection{Quality factor calculation of microcantilever ARROW waveguide}

Quality factor for the microcantilever ARROW B waveguide is analyzed. Quality factor or Q-factor is the ratio of resonant wavelength to full width half maximum (FWHM) and Q-factor is depicted in the equation (11). The electromagnetic (EM) wave is involved in the mathematical modelling of ARROW B cantilever waveguide. The 2.5D FDTD simulation is having the EM field in all region of operation. Since field is not decaying completely in the FDTD modeling the quality factor is calculated using slope of the decaying signal using the equation (12) shown. 


$$
\begin{aligned}
& Q=\frac{\omega_{r}}{F W H M} \\
& Q=\frac{-\omega_{r} * \log _{10} e}{2 m}
\end{aligned}
$$

In the above equations $\omega_{r}$ is the resonant frequency of the decaying signal. $\mathrm{m}$ is the slope of the signal. The simulation is conducted by varying the gap distance between the output waveguide and cantilever i.e., air gap distance. The reported Q-factor also called as quality factor are comparable with the result obtained in article [12]. Q-factor obtained for the air gap distance of $2 \mu \mathrm{m}$ is 15.10 . The gap distance and its corresponding Q-factor and wavelength values are tabulated in the table 3 .

Table 3. Q-factor for cantilever ARROW-B.

\begin{tabular}{ccc}
\hline Gap distance & Q-factor & Wavelength in $\mathbf{~ m m}$ \\
\hline 2 & 15.10 & 506.932 \\
5 & 14.23 & 500.002 \\
6 & 13.31 & 498.251 \\
7 & 12.24 & 496.231 \\
\hline
\end{tabular}

The graph of the Q-factor and wavelength for different values of the air gap distance is shown in figure 12.

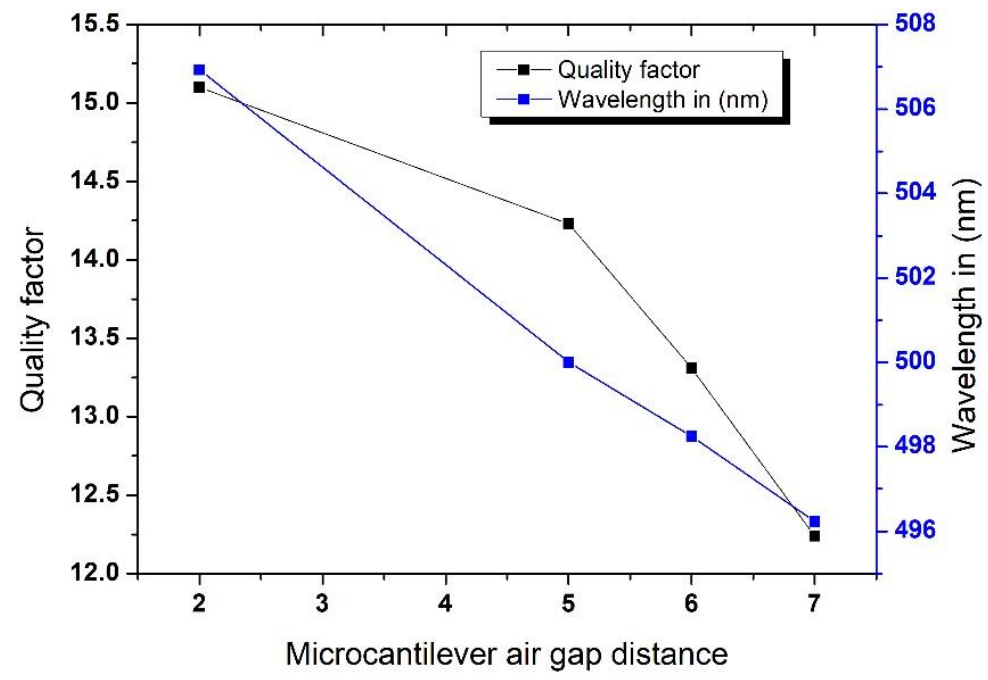

Figure 12. Quality factor and wavelength variation with gap distance.

\section{Discussion}

In this section we discuss the light intensity analysis of the ARROW-B microcantilever waveguide with respect to its $\mathrm{X}$-axis and also sensitivity analysis of the device modeled.

\subsection{Intensity Analysis}

Electric field intensity for the cantilever beam in the ARROW waveguide is modeled and analyzed. Power and field monitors are used to find the E-field intensity. The light source used in the simulation process is in the spectral range from $400 \mathrm{~nm}$ to $700 \mathrm{~nm}$. The spectrum of the light source used is shown in the figure 13. Modal analysis of the ARROW microcantilever waveguide is carried at wavelength $632.8 \mathrm{~nm}$ in the visible spectrum. 

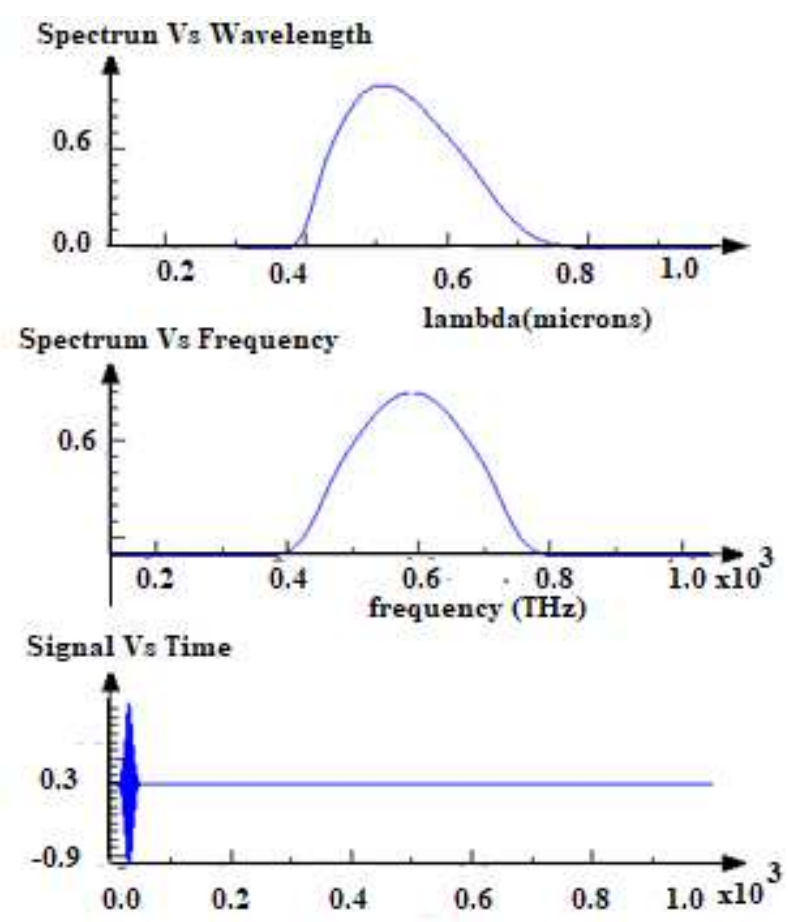

Figure 13. Photonic light wave source

The figure 14 shows the electric field intensity curve of the cantilever waveguide region. The measured intensity is at the input cantilever waveguide region and at the output waveguide. The power monitors are used to obtain the intensity spectrum. The 2.5D or variational FDTD is the method used for modeling the ARROW B cantilever waveguide. The power monitor gives the electric field, magnetic field and pointing vector details. The perfectly matched layer boundary conditions are used to minimize the light absorbance from the device.

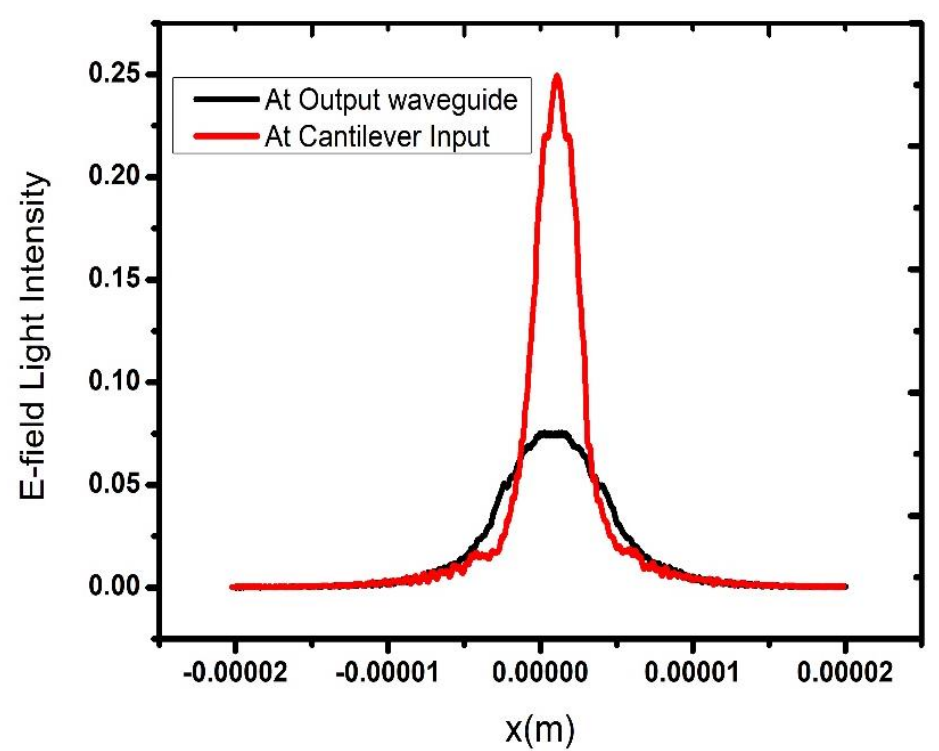

Figure 14. Electric field intensity

The intensity of the light decreases when it is propagating along the cantilever structure and mathematically is represented by the equation (13)

$$
P(x)=P_{0} \exp (-\alpha x)
$$

In equation (13) the $\alpha$ absorption parameter, $P_{0}$ is the power given to the cantilever $(\mathrm{x}=0)$. The output power analysis of the presented work satisfies the above power analysis equation.

\subsection{Sensitivity}


The designed model is simulated by considering the surrounding material as air and also as liquid. When fluid is used for simulation such as blood sample with refractive index 1.33 and hemoglobin refractive index is 1.3854 [31]. The behavior of the system is discussed in this section along with sensitivity. Flow of fluid in the waveguide is laminar and Newtonian and it satisfies the Navier stokes equation (14) as shown depicted below [12].

$$
\rho \frac{\mathrm{dv}}{\mathrm{dt}}+\rho(\mathrm{v} \cdot \nabla) \mathrm{v}=-\nabla \mathrm{p}+\mu \nabla^{2} \mathrm{v}
$$

Here, the density is $\rho$ and velocity is $\mathrm{v}$ and pressure is represented as $\mathrm{p}$. Viscosity of the fluid is $\mu$. The Reynolds number of the device is given by equation (15).

$$
R e=\frac{|\rho \partial v / \partial t|}{4 \cdot\left|\mu \nabla^{2} v\right|}=\frac{\rho \omega b^{2}}{4 \mu}
$$

The simulation is carried out for both air and blood as surrounding material. The fluid flow modeling is simulated with the design is Newtonians and equation (14) and (15) are used in the simulation of the fluid interaction. The sensitivity of the sensor is the ratio of change in the wavelength with respect change in the refractive index of the analyte used. The sensitivity is given by equation (16). The reported sensitivity for different analyte refractive index is given in table 4 . The average sensitivity with our work is $73.78 \mathrm{~nm} / \mathrm{RIU}$. The obtained sensitivity is comparable with the sensitivity values obtained in article [5]. The variation in the refractive index of the material is denoted as refractive index unit (RIU). The figure 15 shows wavelength versus refractive index plot for the different analyte concentration.

$$
S=\frac{\Delta \lambda}{\Delta n}
$$

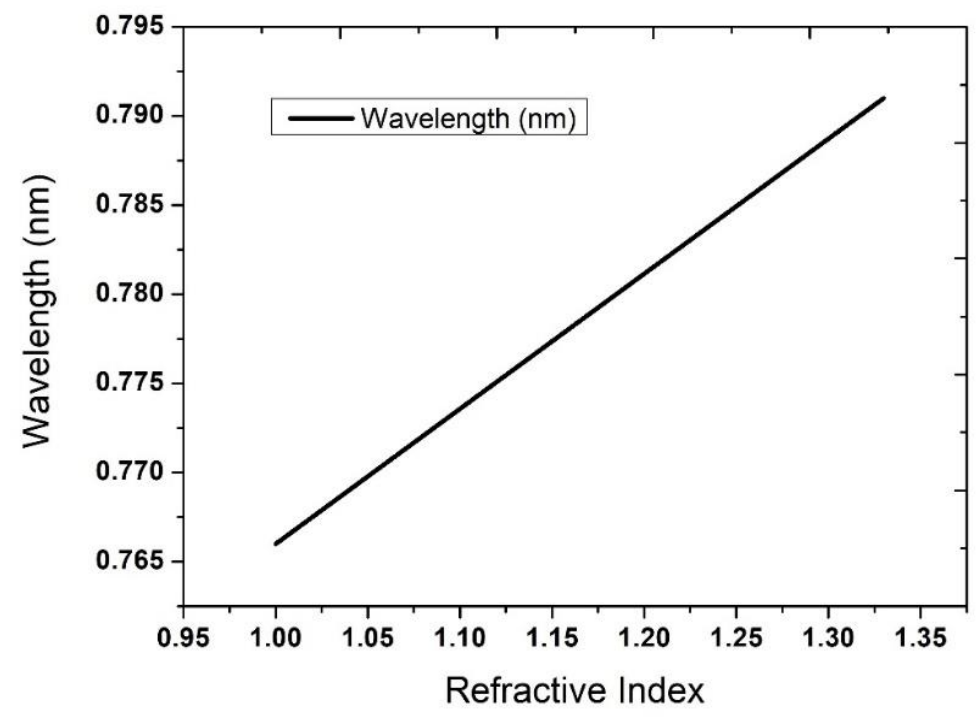

Figure 15. Refractive index-wavelength plot.

Sensitivity is the main design parameter while designing sensor. The designed and simulated ARROW microcantilever waveguide can be used as refractive index-based sensor in medical application. The air gap distance of one micron is considered while designing the sensitivity analysis. The variation of the sensitivity upon changing the refractive index of the surrounding fluid are listed in table 4 . The change in RI versus sensitivity plot is depicted in figure 16 .

Table 4. Sensitivity values for RI variation.

\begin{tabular}{ccccc}
\hline SL NO & RI & Q-factor & $\begin{array}{c}\text { Wavelength in } \\
\mathbf{n m}\end{array}$ & $\begin{array}{c}\text { Sensitivity } \\
\text { (nm/RIU) }\end{array}$ \\
\hline 1 & 1 & 25.183 & 766 & 75.75 \\
2 & 1.33 & 24.257 & 791 & 71.81 \\
3 & $1.3857^{*}$ & 24.048 & 795 & -- \\
\hline
\end{tabular}




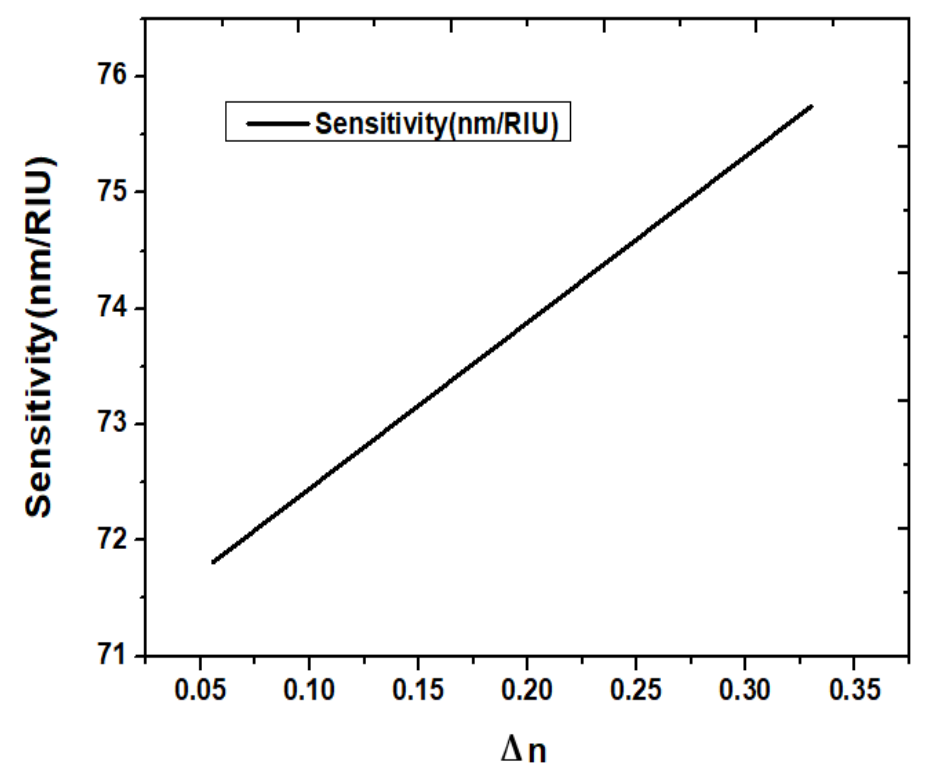

Figure 16. Sensitivity versus refractive index change.

\section{Conclusions}

The simulation and modeling of the microcantilever beam and microcantilever ARROW waveguide has been presented. The designed ARROW microcantilever waveguide is a refractive index-based sensor has application in biomedical field. The changes in the refractive index results in change of the wavelength of the device. The length, width and thickness of silicon nitride microcantilever is $300 \times 40 \times 20 \mu \mathrm{m}$ simulated using finite element method. The electrostatic voltage is applied to the cantilever using the electrodes present in the insulated chamber. The designed system acts like a parallel plate capacitor. The changes in the displacement for applied voltage and capacitance are measured. The ARROW microcantilever waveguide is the second structure designed and simulated using visible light. The ARROW microcantilever waveguide has 400x10x10 $\mu \mathrm{m}$ length, thickness and width in dimension. The ARROW microcantilever is simulated and analyzed for different refractive indices values. The sensitivity of the sensor has good response and results are comparable. The main purpose of the ARROW microcantilever waveguide is in the sensing of the analyte sample in lab-on-a-chip application.

Author Contributions: Conceptualization of the work: Asha K; methodology, Asha K.; validation, Asha K., Suryanarayana N K; writing — original draft preparation, Asha K.; writing - review and editing, Suryanarayana N K; supervision, Narayan K; project administration, Narayan K.; funding acquisition, Narayan K.

Funding: "The research was funded by VGST IT, BT and ST GOK, grant number VGST/CSIEE.GRD/466.

Acknowledgments: The authors would like to thank Principal and Management of SVIT, Bangalore and also VTU, Belagavi, Karnataka for inculcating the research culture.

Conflicts of Interest: "The authors declare no conflict of interest."

\section{Availability of data and material \\ Not Applicable}

\section{Code availability}

Not Applicable

\section{References}

1. Ansari, Mohd Zahid, and Chongdu Cho. "Design and analysis of a high sensitive microcantilever biosensor for biomedical applications." In 2008 International Conference on BioMedical Engineering and Informatics, vol. 2, pp. 593-597. IEEE, 2008. 
2. Gupta, Raj K. "Electrostatic pull-in test structure design for in-situ mechanical property measurements of microelectromechanical systems (MEMS)." PhD diss., Massachusetts Institute of Technology, 1997.

3. Sader, John Elie. "Frequency response of cantilever beams immersed in viscous fluids with applications to the atomic force microscope." Journal of applied physics 84, no. 1 (1998): 64-76.

4. Agarwal, Pranav, Deepak Sahoo, Abu Sebastian, Haris Pozidis, and Murti V. Salapaka. "Modeling and identification of the dynamics of electrostatically actuated microcantilever with integrated thermal sensor." In 2008 47th IEEE Conference on Decision and Control, pp. 2624-2630. IEEE, 2008.

5. Jing, Yachao, Guofang Fan, Rongwei Wang, Zeping Zhang, Muguang Wang, Xiaoyu Cai, Jiasi Wei, Xin Chen, Hongyu Li, and Yuan Li. "Analysis for an Improved Nanomechanical Microcantilever Sensor on Optical Waveguides." IEEE Access 8 (2020): 63856-63861.

6. Boisen, Anja, Jacob Thaysen, Henriette Jensenius, and Ole Hansen. "Environmental sensors based on micromachined cantilevers with integrated read-out." Ultramicroscopy 82, no. 1-4 (2000): 11-16.

7. Caruntu, Dumitru I., and Israel Martinez. "Reduced order model of parametric resonance of electrostatically actuated MEMS cantilever resonators." International Journal of Non-Linear Mechanics 66 (2014): 28-32.

8. Chaterjee, S., and G. Pohit. "A large deflection model for the pull-in analysis of electrostatically actuated microcantilever beams." Journal of sound and vibration 322, no. 4-5 (2009): 969-986.

9. Gajula, Durga, Ferhat Bayram, Ifat Jahangir, Digangana Khan, and Goutam Koley. "Dynamic response of VO 2 mesa based GaN microcantilevers for sensing applications." In 2019 IEEE SENSORS, pp. 1-4. IEEE, 2019.

10. Green, Christopher P., and John E. Sader. "Torsional frequency response of cantilever beams immersed in viscous fluids with applications to the atomic force microscope." Journal of applied physics 92, no. 10 (2002): 6262-6274.

11. Plaza, José Antonio, Kirill Zinoviev, G. Villanueva, Mar Álvarez, J. Tamayo, Carlos Domínguez, and Laura M. Lechuga. "T-shaped microcantilever sensor with reduced deflection offset." Applied physics letters 89, no. 9 (2006): 094109.

12. Basak, Sudipta, Arvind Raman, and Suresh V. Garimella. "Hydrodynamic loading of microcantilevers vibrating in viscous fluids." Journal of Applied Physics 99, no. 11 (2006): 114906.

13. Nezhad, Amir Sanati, Mahmood Ghanbari, Carlos G. Agudelo, Muthukumaran Packirisamy, Rama B. Bhat, and Anja Geitmann. "PDMS microcantilever-based flow sensor integration for lab-on-a-chip." IEEE Sensors journal 13, no. 2 (2012): 601-609.

14. Nezhad, Amir Sanati, Mahmoud Ghanbari, Carlos Gustavo Agudelo, Muthu Packirisamy, and Rama Bhat. "Simulation of PDMS microcantilever deflection using integrated optical fibers." In Photonics North 2011, vol. 8007, p. 80071R. International Society for Optics and Photonics, 2011.

15. Sarwar, Azeem, Petros G. Voulgaris, and Srinivasa M. Salapaka. "Modeling and distributed control of an electrostatically actuated microcantilever array." In 2007 American Control Conference, pp. 4240-4245. IEEE, 2007.

16. Zhang, X. Richard, and Xianfan Xu. "Laser bending for high-precision curvature adjustment of microcantilevers." Applied Physics Letters 86, no. 2 (2005): 021114.

17. Zhou, Wei, Abdul Khaliq, Yanjun Tang, Haifeng Ji, and Rastko R. Selmic. "Simulation and design of piezoelectric microcantilever chemical sensors." Sensors and actuators A: Physical 125, no. 1 (2005): 6975.

18. Zinoviev, Kirill, Carlos Dominguez, Jose Antonio Plaza, Víctor Javier Cadarso Busto, and Laura M. Lechuga. "A novel optical waveguide microcantilever sensor for the detection of nanomechanical forces." Journal of Lightwave Technology 24, no. 5 (2006): 2132.

19. Zinoviev, Kirill, Carlos Dominguez, Jose Antonio Plaza, and Laura M. Lechuga. "Optical waveguide cantilever actuated by light." Applied Physics Letters 92, no. 1 (2008): 011908.

20. Hu, Yuh-Chung, C. M. Chang, and S. C. Huang. "Some design considerations on the electrostatically actuated microstructures." Sensors and Actuators A: Physical 112, no. 1 (2004): 155-161.

21. Mehdipour, Iman, Ahmad Erfani-Moghadam, and Cyrus Mehdipour. "Application of an electrostatically actuated cantilevered carbon nanotube with an attached mass as a bio-mass sensor." Current Applied Physics 13, no. 7 (2013): 1463-1469.

22. Ji, Hai-Feng, K. M. Hansen, Z. Hu, and T. Thundat. "Detection of $\mathrm{pH}$ variation using modified microcantilever sensors." Sensors and Actuators B: Chemical 72, no. 3 (2001): 233-238. 
23. Mishra, Rohit, Wilfried Grange, and Martin Hegner. "Rapid and reliable calibration of laser beam deflection system for microcantilever-based sensor setups." Journal of Sensors 2012 (2012).

24. Kwon, Beomjin, Myunghoon Seong, Jui-Nung Liu, Matthew R. Rosenberger, Matthew V. Schulmerich, Rohit Bhargava, Brian T. Cunningham, and William P. King. "Large infrared absorptance of bimaterial microcantilevers based on silicon high contrast grating." Journal of Applied Physics 114, no. 15 (2013): 153511.

25. Lavrik, Nickolay V., and Panos G. Datskos. "Femtogram mass detection using photothermally actuated nanomechanical resonators." Applied physics letters 82, no. 16 (2003): 2697-2699.

26. Mishra, Neha K., and Bharatbhushan S. Kale. "Fabrication of tapered and conductive microcantilever." In 2017 International Conference on Nascent Technologies in Engineering (ICNTE), pp. 1-4. IEEE, 2017.

27. Moulin, A. M., S. J. O'shea, and Mark E. Welland. "Microcantilever-based biosensors." Ultramicroscopy 82, no. 1-4 (2000): 23-31.

28. Shang-Rou, Hsieh, Steven W. Shaw, and Christophe Pierre. "Normal modes for large amplitude vibration of a cantilever beam." International Journal of Solids and Structures 31, no. 14 (1994): 1981-2014.

29. M. Nordström, D. A. Zauner, "Integrated optical readout for miniaturization of cantilever-based sensor system”, Applied physics letters, 91, 103512 (2007).

30. Baba, Toshihiko, and Yasuo Kokubun. "Dispersion and radiation loss characteristics of antiresonant reflecting optical waveguides-numerical results and analytical expressions." IEEE Journal of Quantum electronics 28, no. 7 (1992): 1689-1700.

31. Lazareva, Ekaterina N., and Valery V. Tuchin. "Blood refractive index modelling in the visible and near infrared spectral regions." Journal of Biomedical Photonics \& Engineering 4, no. 1 (2018). 
Figures

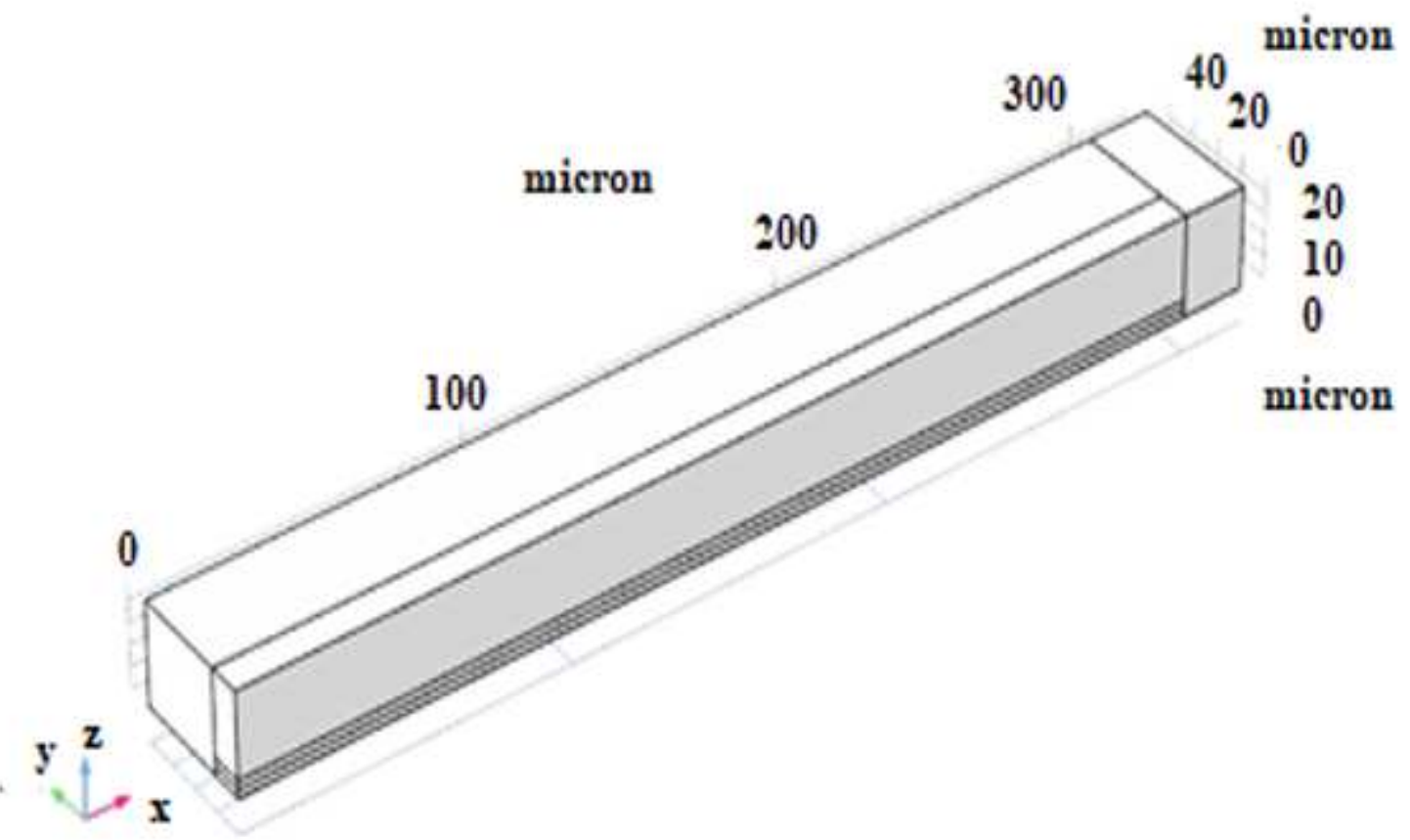

Figure 1

Microcantilever Beam

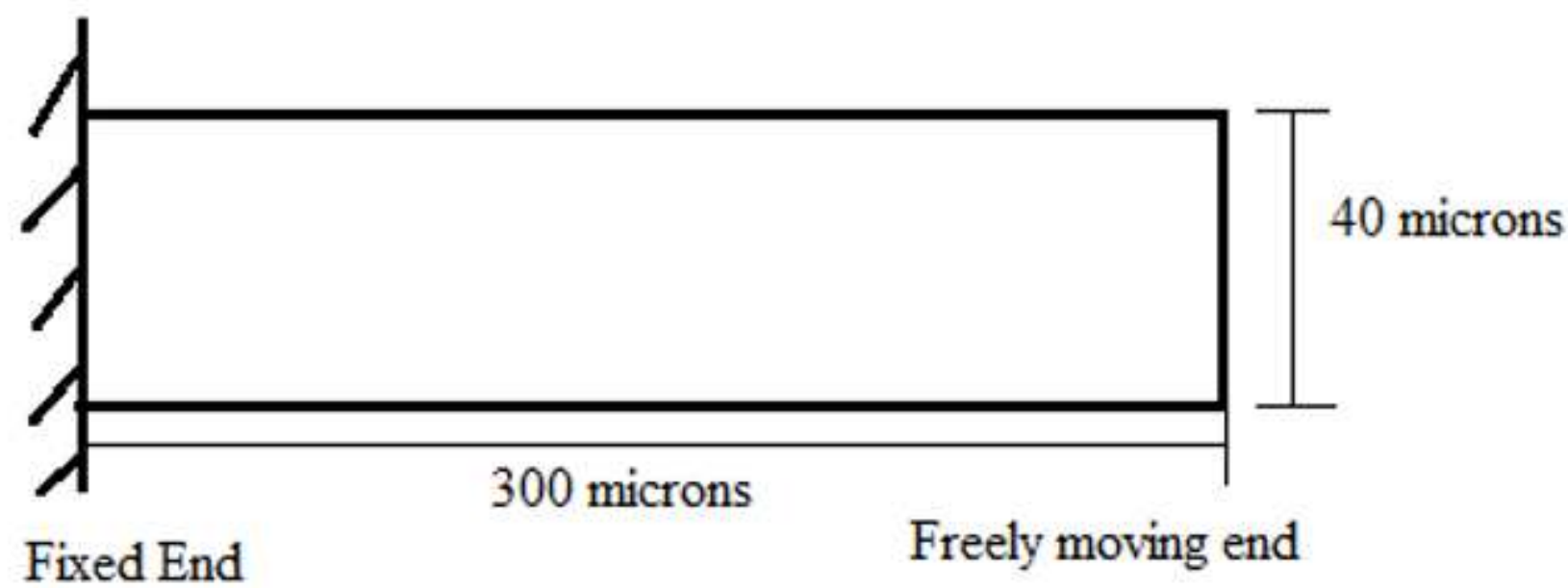

Figure 2

Two-dimensional view of the microcantilever beam 


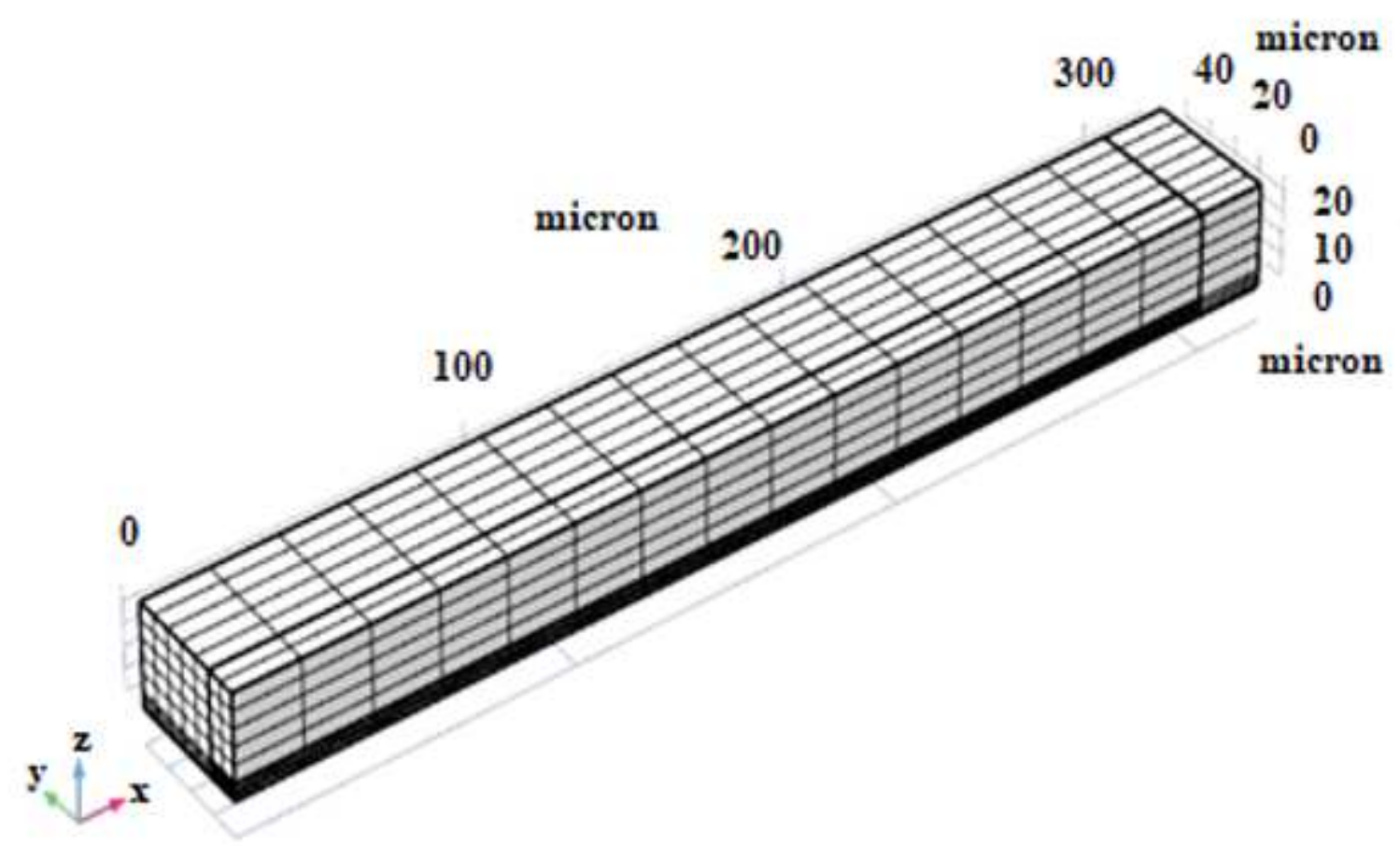

Figure 3

Meshing of microcantilever beam

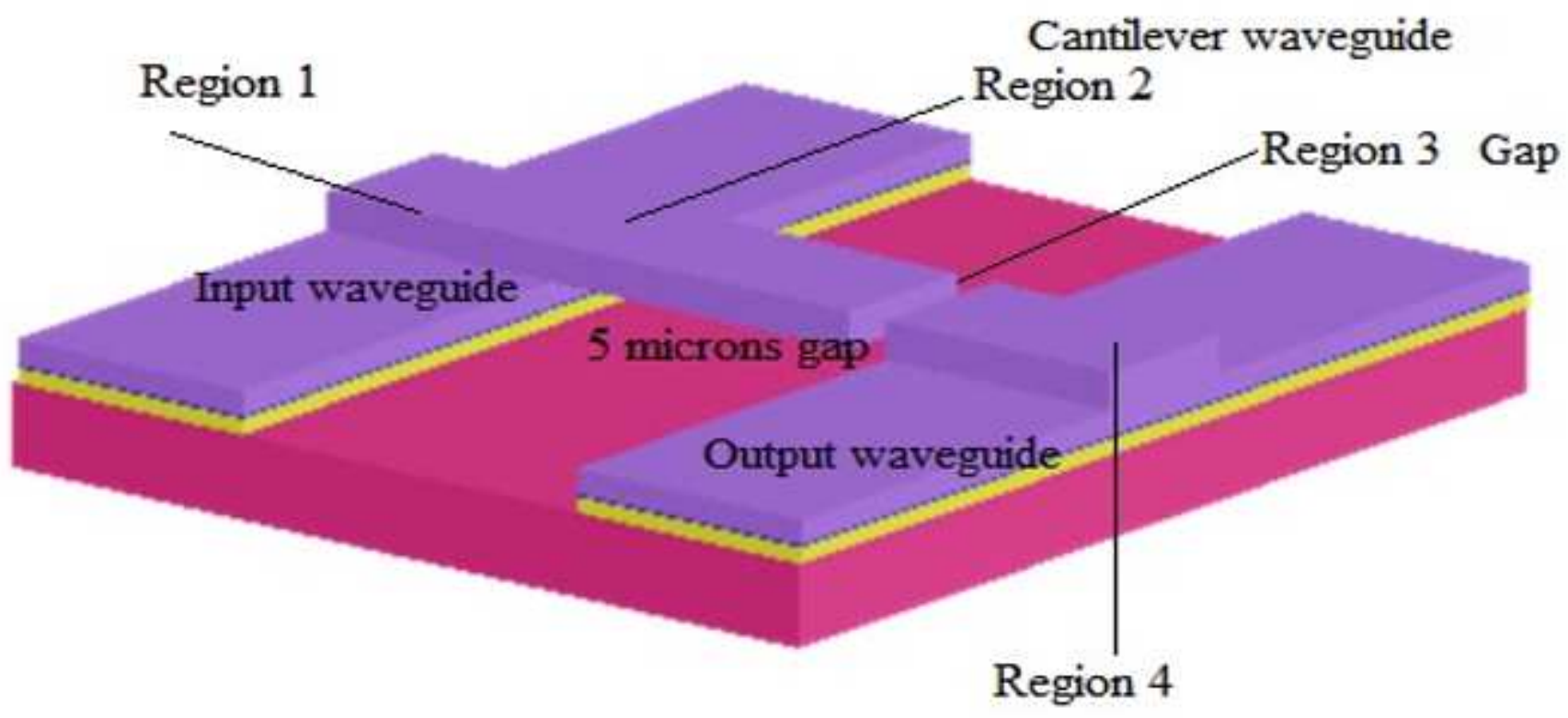

Figure 4

Microcantilever ARROW device 


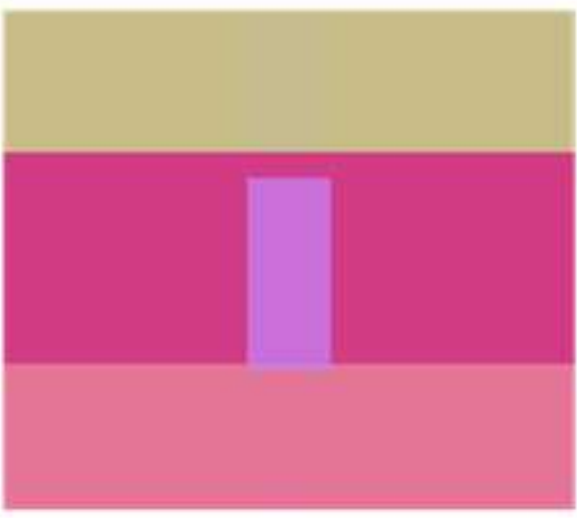

(a) $\mathrm{XY}$ view

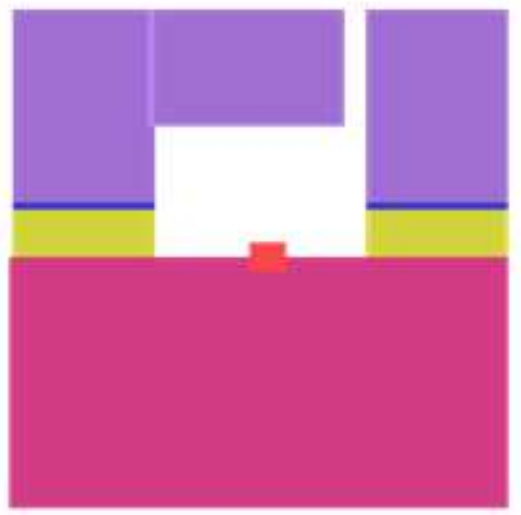

(c)YZ view

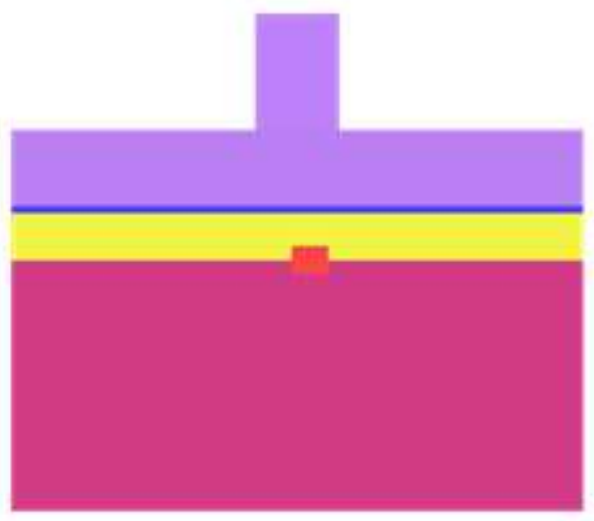

(b) $\mathrm{XZ}$ view

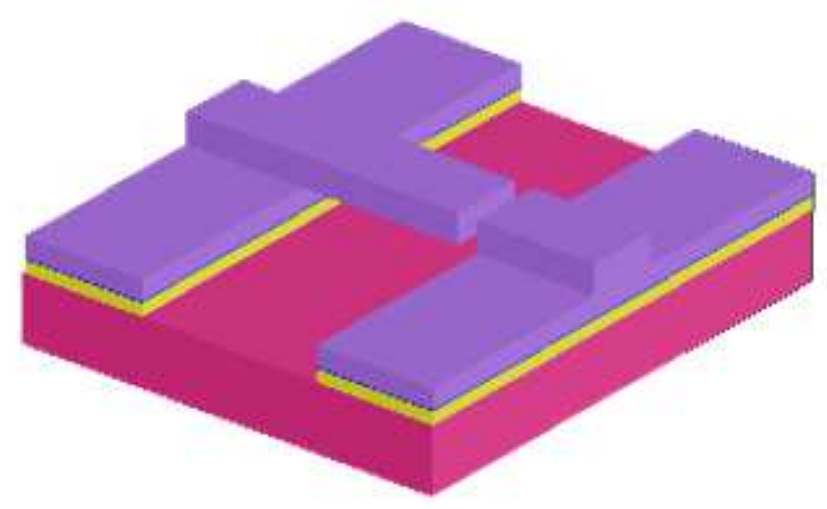

(d) Complete view of microcantilever

Figure 5

Directional view of Microcantilever ARROW device 
Region 4

Output ARROW-B

Waveguide

$$
\mathrm{n}=1.00
$$

\begin{tabular}{|c|}
\hline$n_{\text {core }}$ \\
\hline$n_{\mathrm{fcl}}$ \\
\hline $\mathrm{n}_{\mathrm{scl}}$ \\
\hline \\
$\mathrm{n}_{\mathrm{sub}}$ \\
\hline
\end{tabular}

Region 3

Air Gap

Region 2

Cantilever
Region 1

Input ARROW-B

Waveguide

$$
\mathrm{n}=1.00
$$

\section{Figure 6}

Regions of microcantilever ARROW waveguide

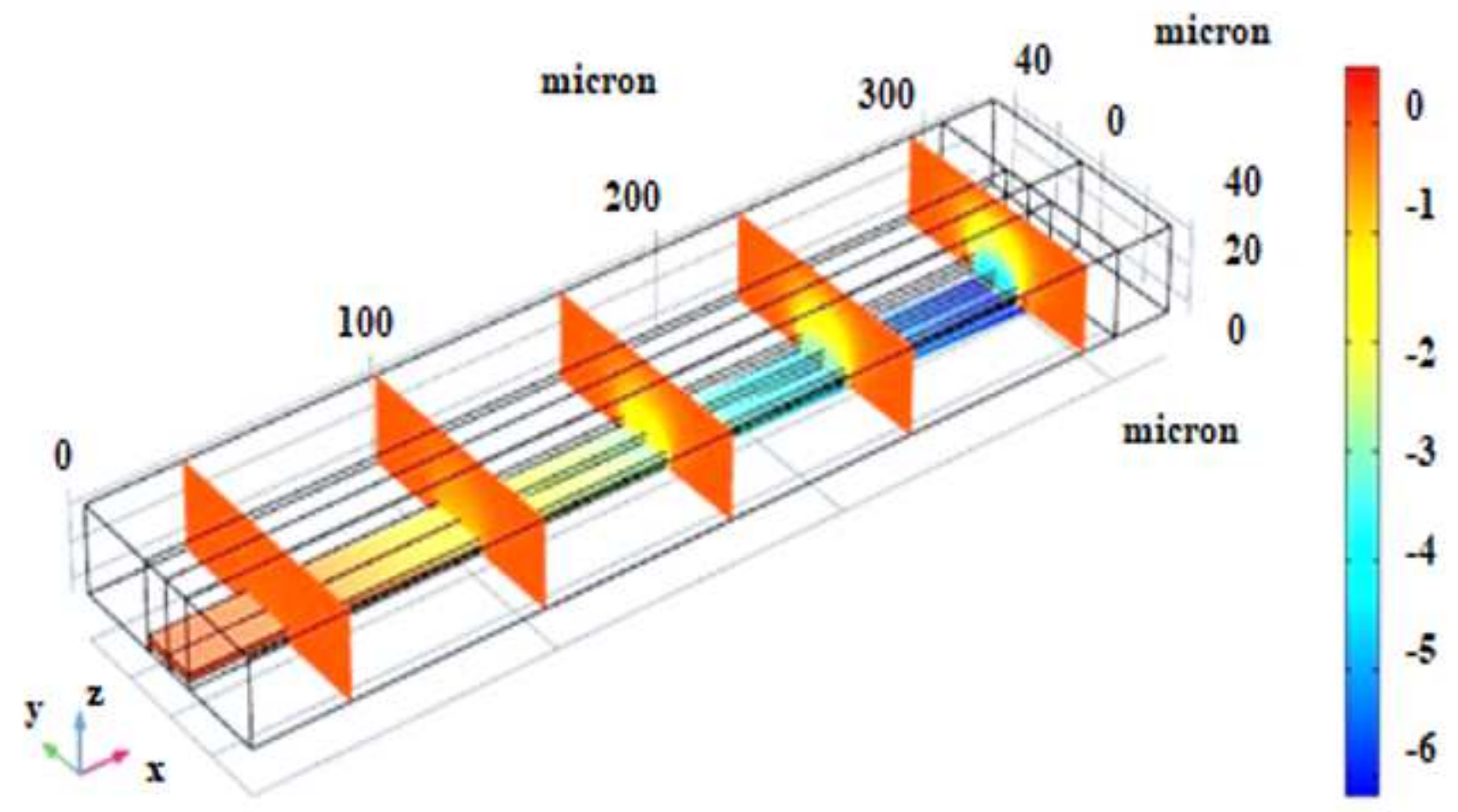

Figure 7

Displacement of the cantilever 


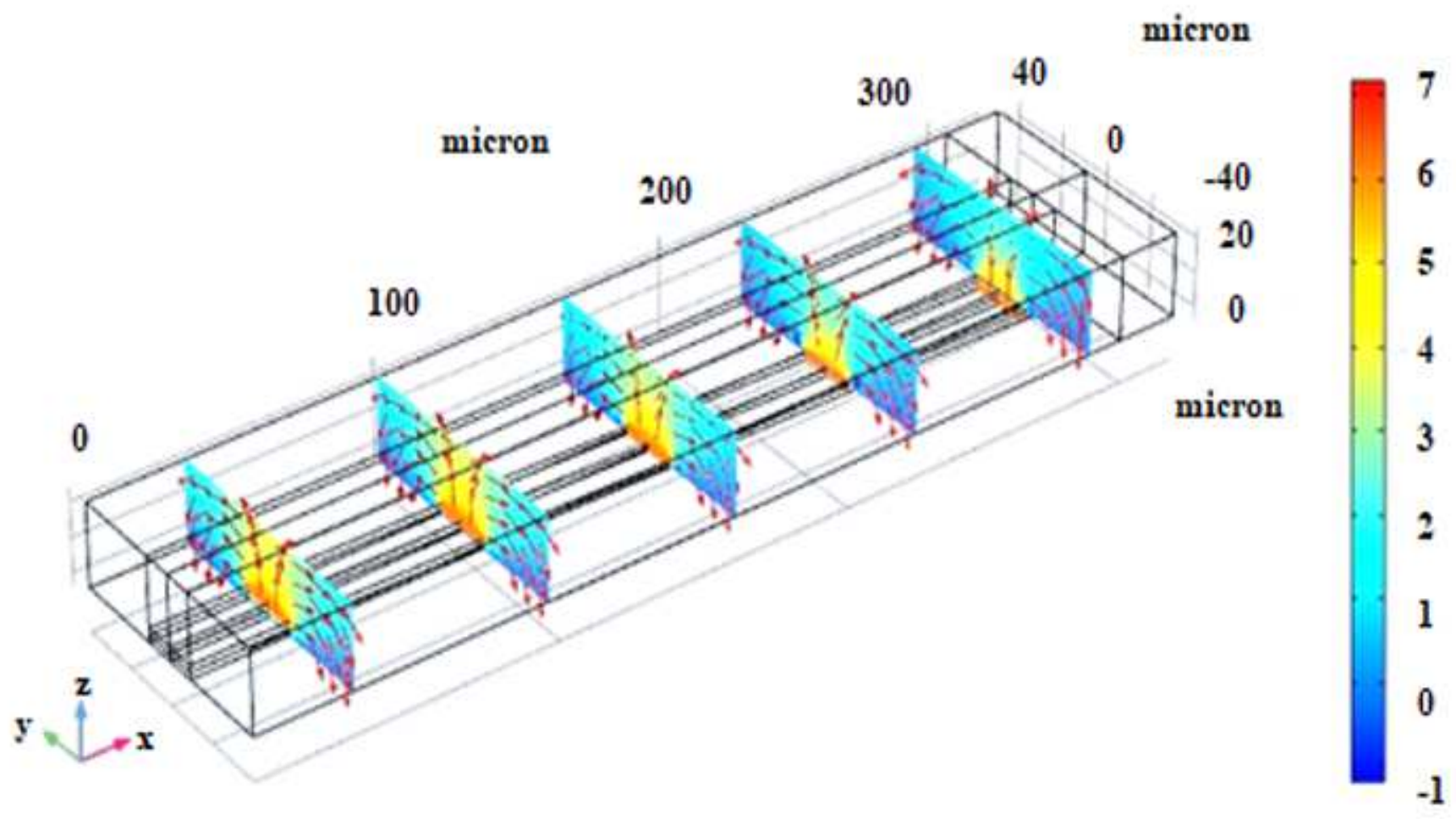

Figure 8

Electric field and potential of cantilever beam 
shape of the cantilever displacement for different applied voltage

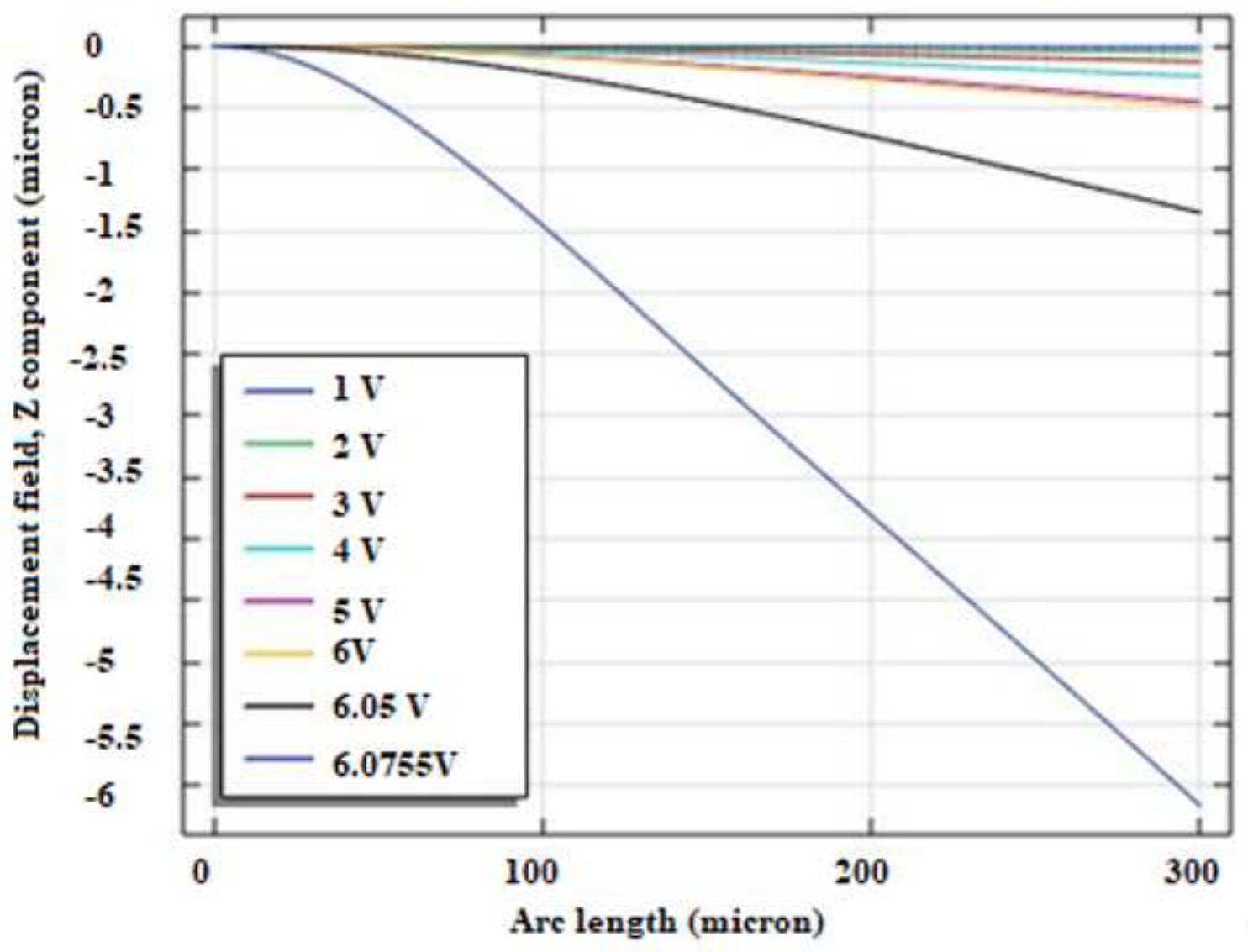

Figure 9

Cantilever Displacement versus arc length. 


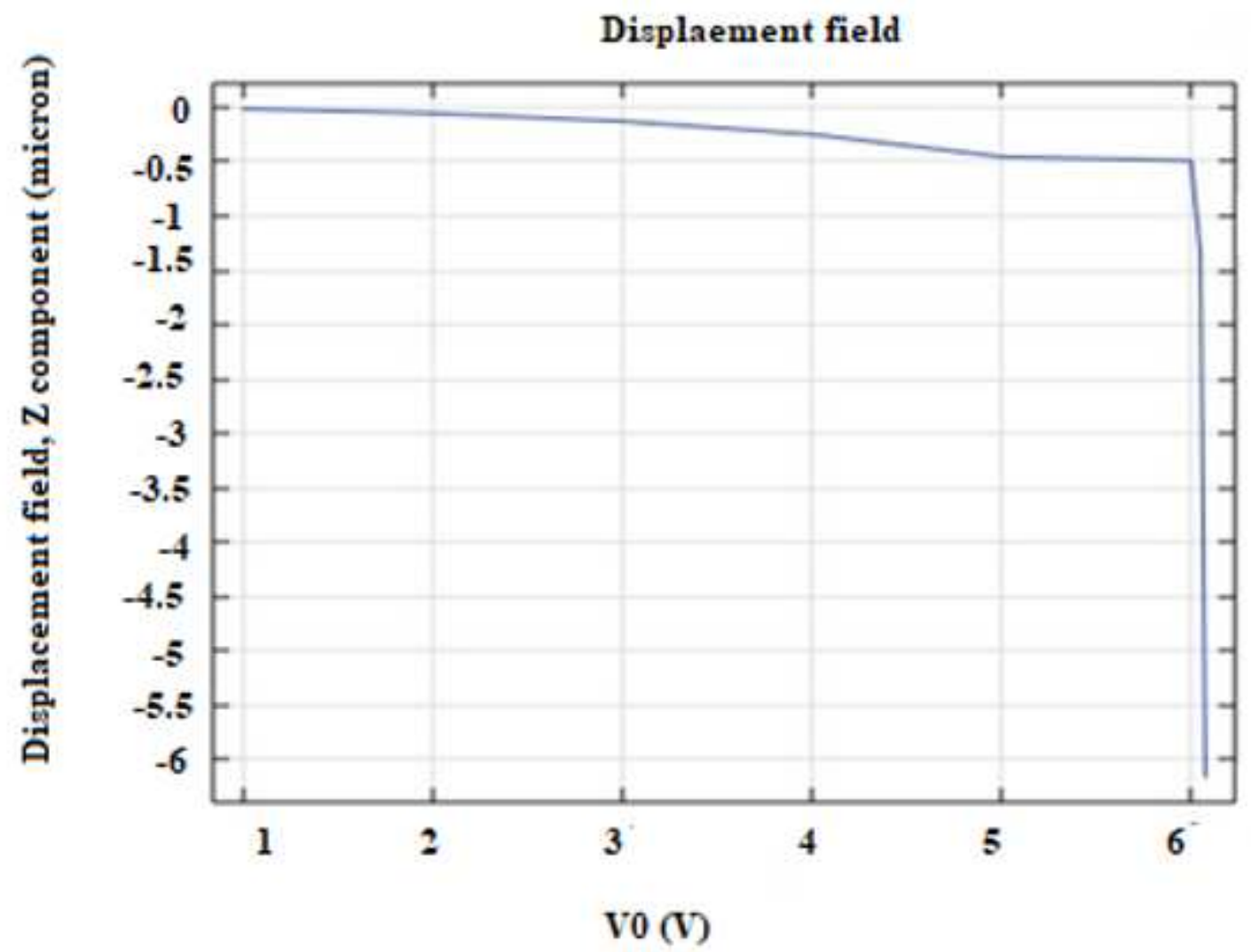

Figure 10

Electrical voltage applied versus Displacement. 


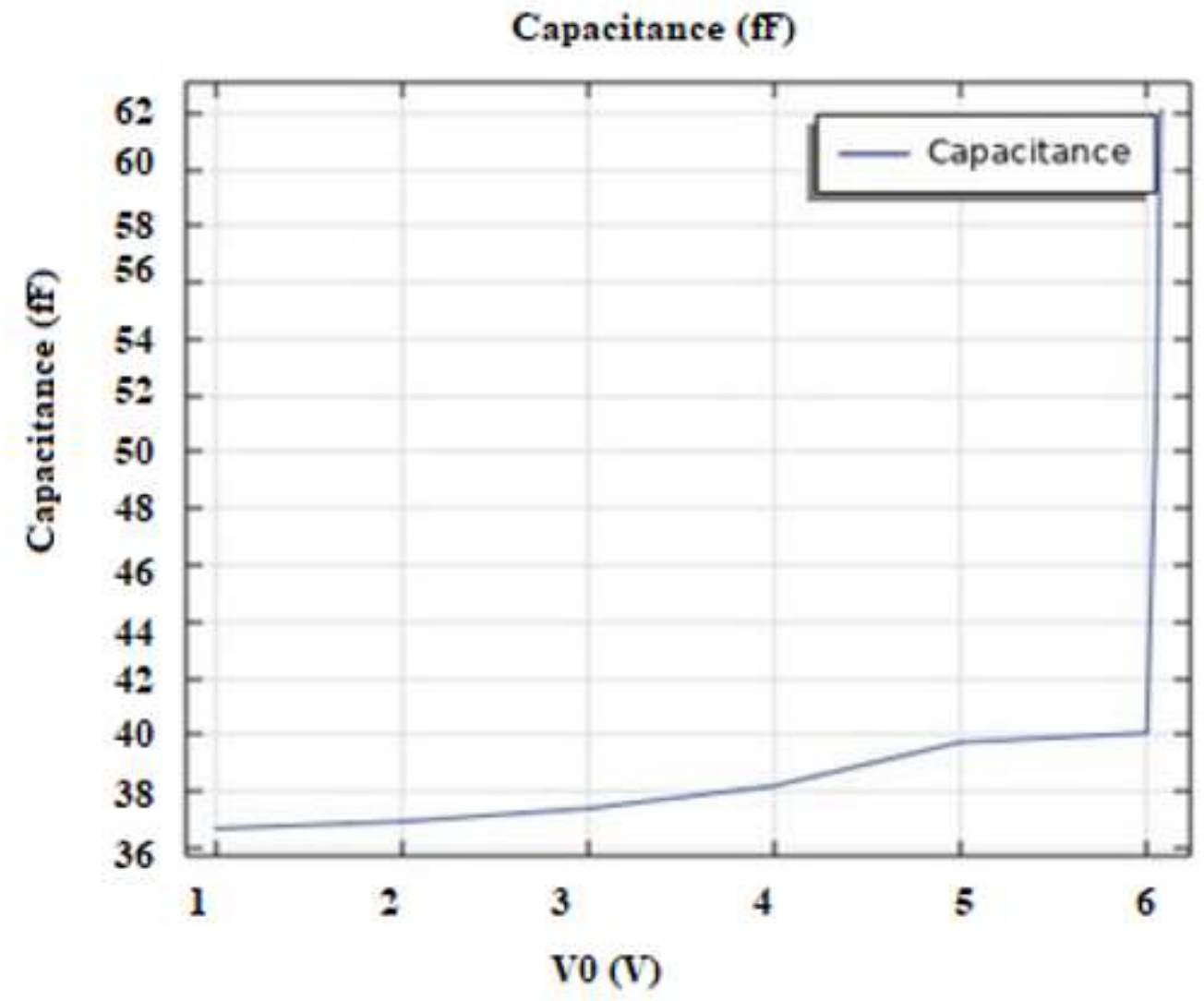

Figure 11

DC capacitance versus voltage applied

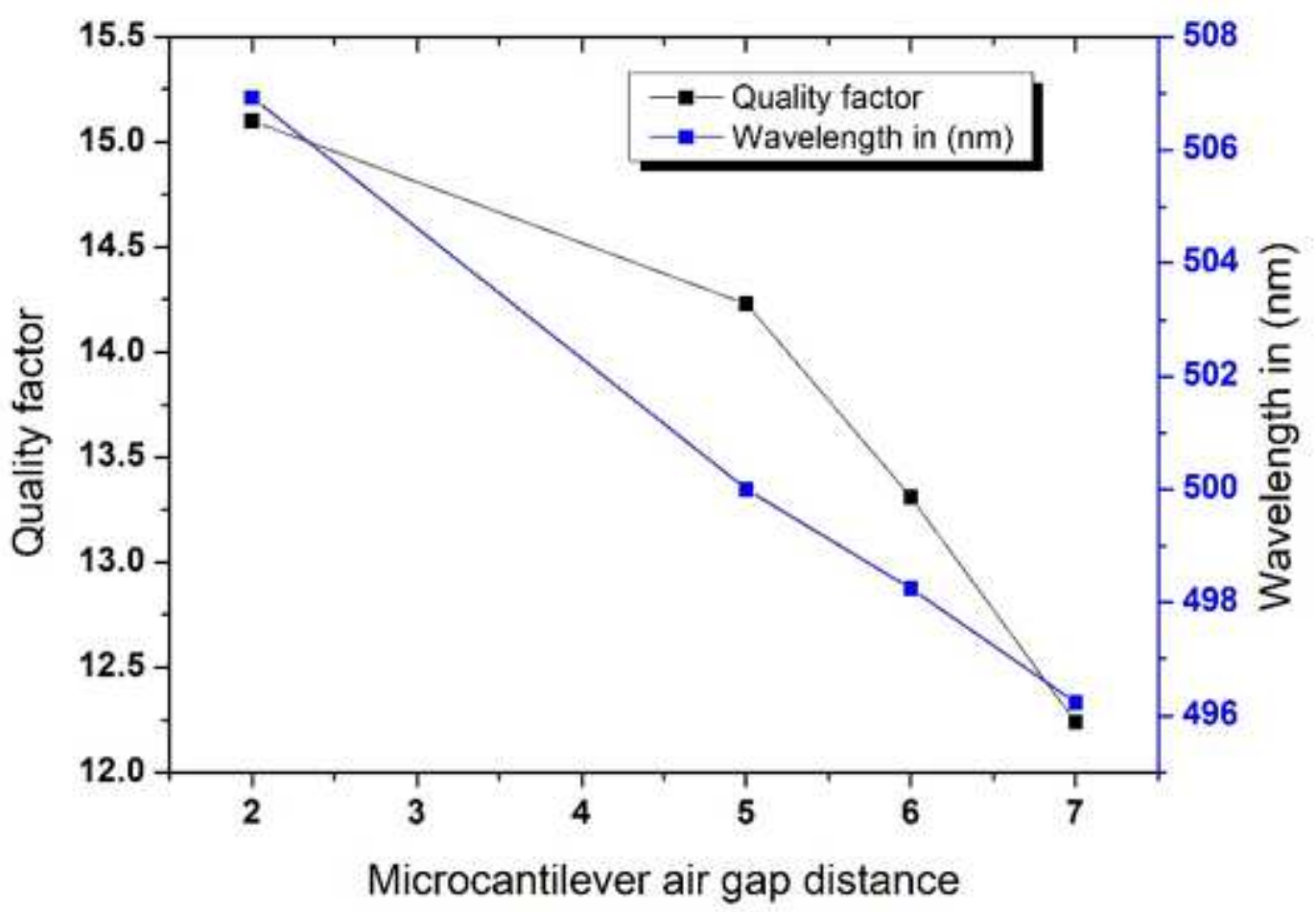


Figure 12

Quality factor and wavelength variation with gap distance.
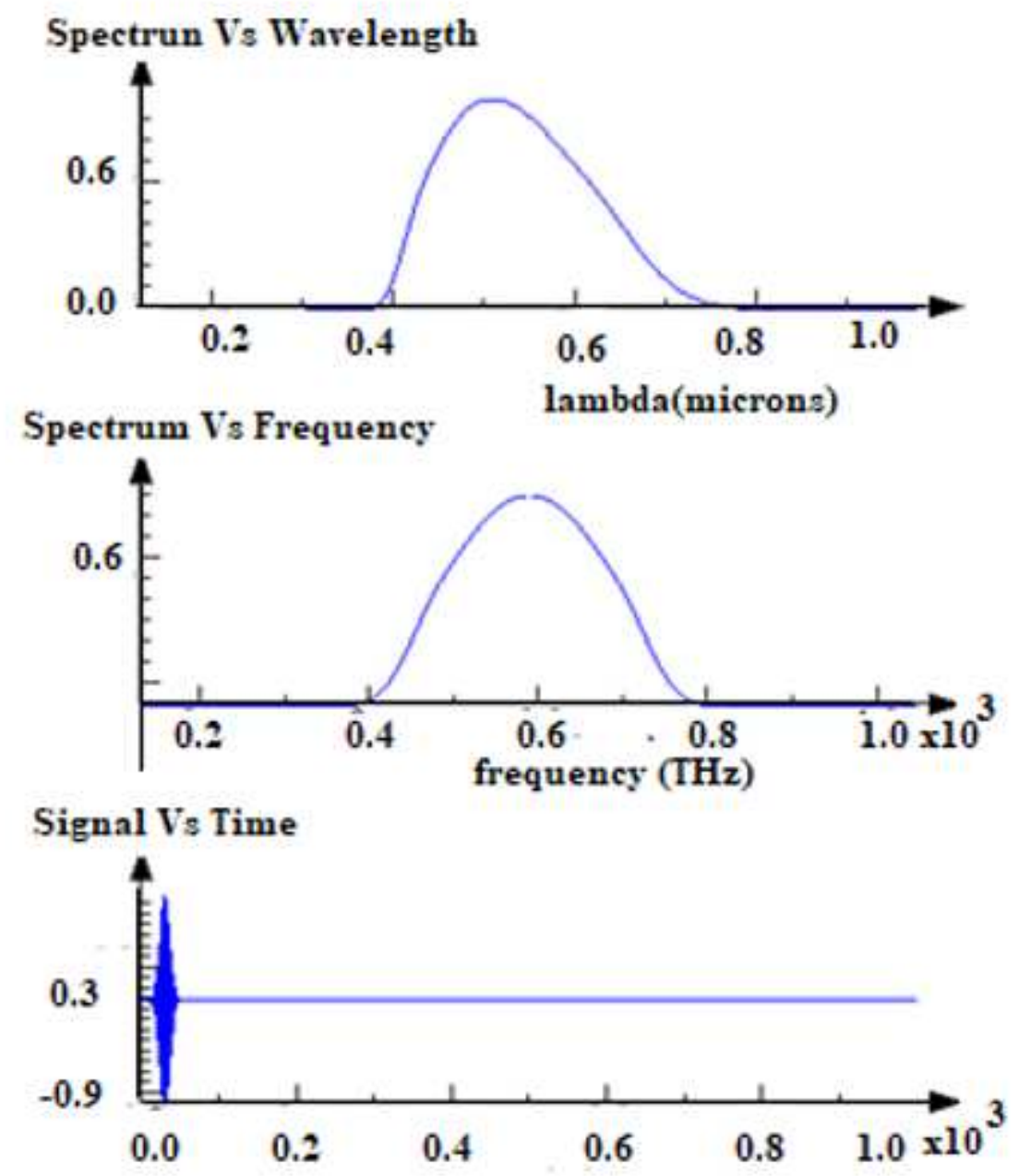

Figure 13

Photonic light wave source 


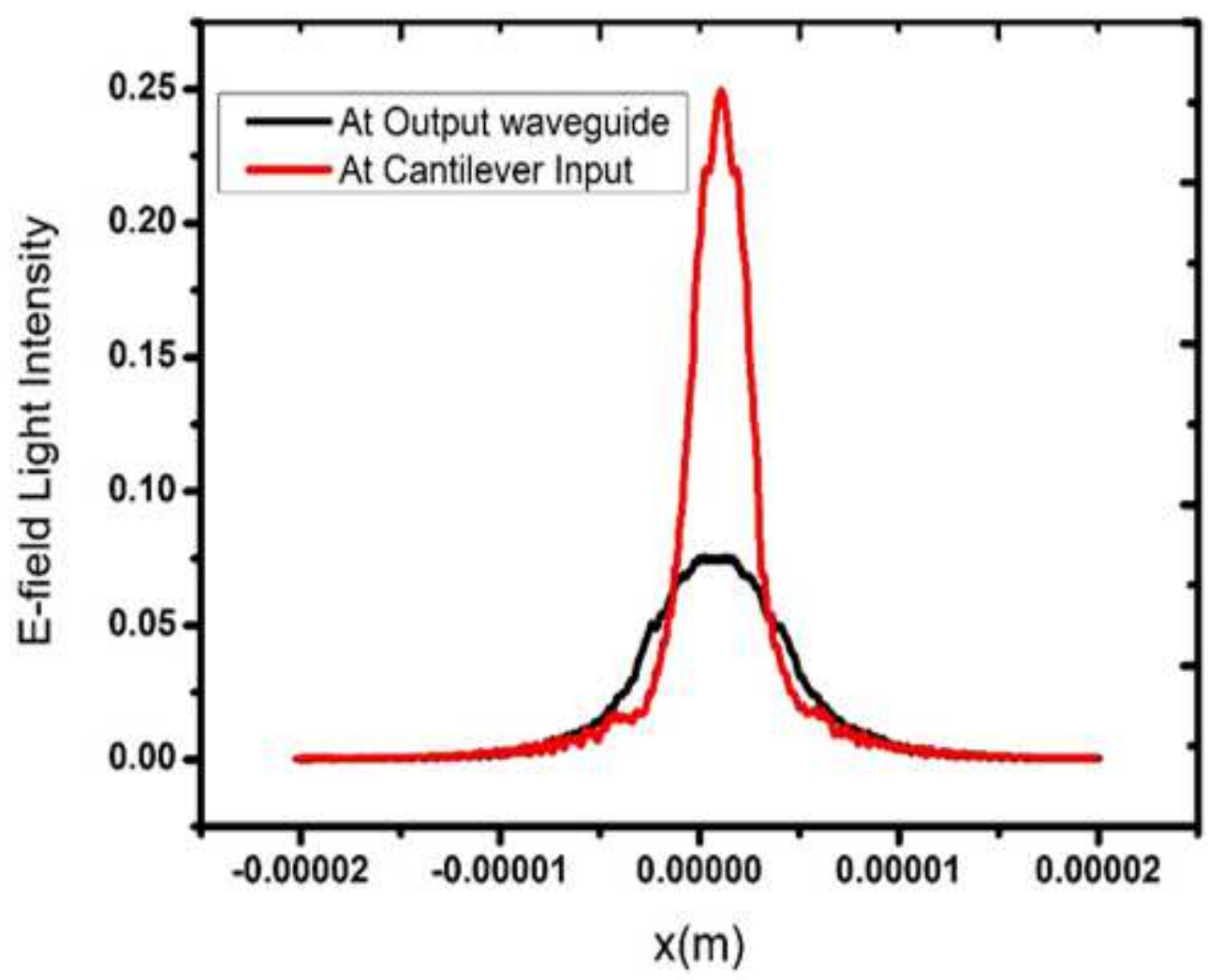

Figure 14

Electric field intensity

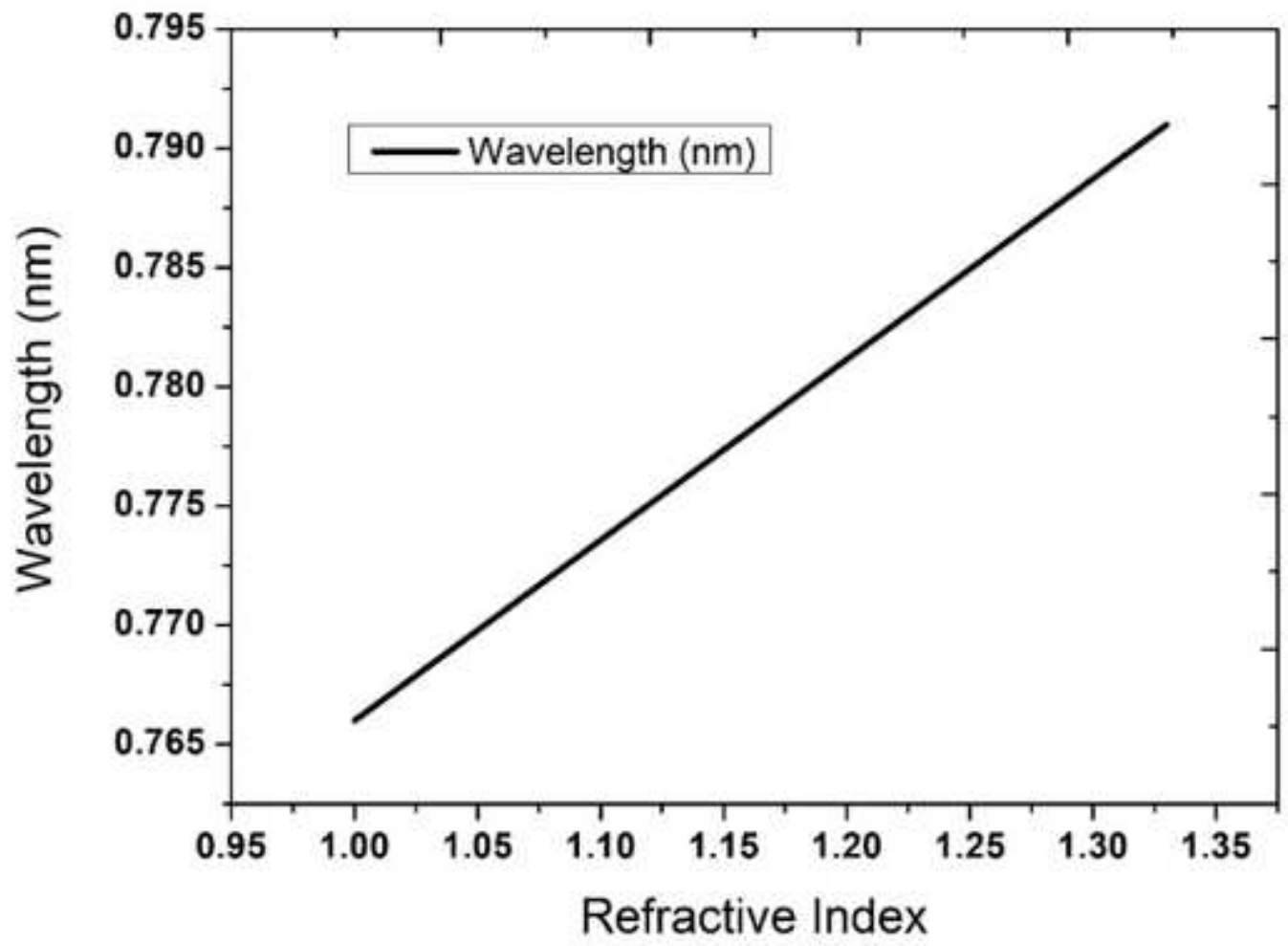


Figure 15

Refractive index-wavelength plot.

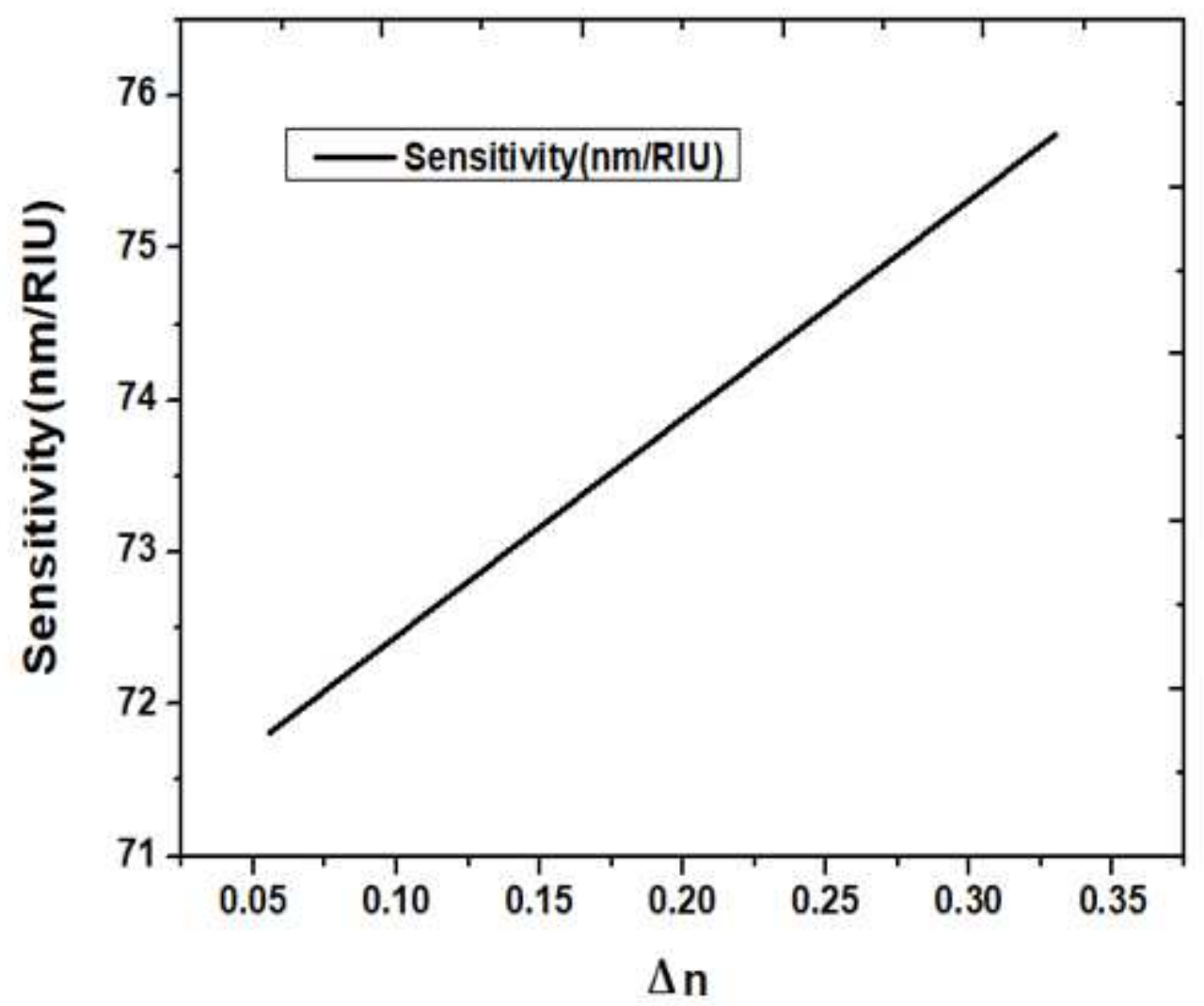

Figure 16

Sensitivity versus refractive index change. 